\title{
Modeling of Wedge-shaped Anisotropic Piezocomposite Transducer for Guided Wave-based Structural Health Monitoring
}

\author{
Ken I. Salas*, Carlos E. S. Cesnikł and Ajay Raghavan ${ }^{\ddagger}$ \\ Department of Aerospace Engineering, The University of Michigan, Ann Arbor, MI, 48109, USA
}

The guided wave (GW) field excited by a wedge-shaped, anisotropic piezocomposite transducer (APT), surface-bonded on an isotropic substrate is investigated with applications to large area structural health monitoring (SHM). It is shown that this configuration is significantly more efficient than conventional designs in terms of electric current drawn to induced displacement amplitudes. The analysis is based on the three-dimensional equations of elasticity, and the solution yields analytical expressions for the field variables in three separate spatial regions determined by the location of the transducer's radial edges. In this study, the dynamics of the substrate and transducer are assumed uncoupled, and their interaction is modeled through surface tractions along the transducer's edges. The excitation modes considered are harmonic and a Hann-modulated toneburst. A similar problem is modeled using three-dimensional finite element simulations, to assess the spatial and transient accuracy of the analytical solution. The results show good agreement over a wide range of radial and azimuthal positions, as well as good time resolution. The resulting expressions are used to identify optimal harmonic excitation frequencies and actuator dimensions. The response of a wedge-shaped APT sensor and a rectangular piezoelectric sensor under the guided wave field excited by a wedge-shaped APT actuator is also investigated.

\section{Nomenclature}

A Area

$A^{*} \quad$ Aspect Ratio

$b \quad$ Substrate half thickness

$\bar{b} \quad$ Residue

C Capacitance

$c_{p}, c_{s}$ Dilatational/Shear wave speed in isotropic material

$c_{k} \quad$ Complex Fourier coefficients of shear tractions

$D \quad$ Dispersion equation for Rayleigh-Lamb waves

$d_{i j} \quad$ Piezoelectric coupling coefficient $(i, j=1,2,3)$

$E, \nu$ Young's modulus and Poisson's ratio

$\overline{\mathbf{F}} \quad$ Shear stresses Fourier transform vector

$g_{i j} \quad$ Piezoelectric constants $(i, j=1,2,3)$

$\mathbf{H}, \varphi$ Helmholtz displacement components

$h, w$ Generic functions

$\widetilde{H}_{k} \quad$ Hankel transform of order $\mathrm{k}$

I Electric current

$J_{m} \quad$ Bessel function of the first kind and order $\mathrm{m}$

\footnotetext{
*Graduate Research Assistant, Student Member, AIAA; Email: ksalas@umich.edu; Ph: 734-936-0541.

${ }^{\dagger}$ Associate Professor and Associate Fellow, AIAA; Corresponding Author; Email: cesnik@umich.edu; Ph: 1-734-764-3397; Fax: 1-734-763-0578.

‡Graduate Research Assistant, Student Member, AIAA; Email: ajayr@umich.edu;
} 
$k \quad$ Dielectric constant

$n \quad$ Number of half-cycles in toneburst signal

$p \quad$ Capacitance function

$r, \theta \quad$ Radial/Azimuthal position

$r^{*} \quad$ Non-dimensional sensor dimension

$\Delta r \quad$ Radial dimension

$R \quad$ Radius

$R_{O} \quad$ Transducer's outer radius

$R_{I} \quad$ Transducer's inner radius

$s(t) \quad$ Toneburst signal

$S(\omega)$ Fourier transform of toneburst signal

$T$ Substrate-actuator thickness ratio

$t$ Time

$\bar{t} \quad$ Thickness

$u($.$) Unit step function$

u Displacement vector

$V \quad$ Voltage

$w^{*} \quad$ Non-dimensional sensor width

$x, y$ Cartesian coordinates

$\boldsymbol{\Gamma}, \boldsymbol{\Psi}$ Coefficient matrices used in analytical solution

$\delta($.$) Dirac delta function$

$\Delta \theta \quad$ Azimuthal dimension

$\epsilon_{i j} \quad$ Strain components $(i, j=x, y$ or $r, \theta)$

$\epsilon_{0} \quad$ Permittivity constant

$\varepsilon \quad$ Error between function and Fourier series representation

$\theta^{*} \quad$ Non-dimensional sensor azimuthal span

$\theta_{L} \quad$ Transducer's left azimuthal edge

$\theta_{R} \quad$ Transducer's right azimuthal edge

$\lambda, \mu \quad$ Substrate Lame constants

$\xi \quad$ Radial wavenumber

$\xi_{x}, \xi_{y}$ Wavenumber along x- and y- directions

$\rho \quad$ Substrate material density

$\tau_{0} \quad$ Force per unit length

$\sigma \quad$ Stress tensor

$\phi \quad$ Azimuthal wavenumber

$\psi \quad$ Substrate-actuator stiffness-thickness ratio

$\omega \quad$ Angular frequency

$\omega_{0} \quad$ Center frequency of toneburst signal

Subscript

a Actuator

$A \quad$ Antisymmetric mode

$B$ Substrate

$P \quad$ Piezoelectric

$s \quad$ Sensor

$S \quad$ Symmetric mode

uc Unit cell

\section{Introduction}

Structural health monitoring (SHM) is an integral part of damage prognosis systems. The primary function of this component is to inspect the condition of a structural element to detect damage and record its evolution in time. This task is usually achieved using a network of transducers distributed around the structure. These transducers are connected to a central processing unit that interprets the data received to detect and identify defects, if present. GW testing methods have gained importance in this area primarily 
because of the ability of guided waves to be transmitted over long distances with little attenuation. ${ }^{1}$ The wave field necessary for damage detection using GW SHM is usually excited using piezoelectric transducers, with the most common transducer type being simple piezoelectric wafers (hereafter referred to as piezos) bonded on the surface of the structure to be inspected. Typical materials used for the construction of these wafers include lead zirconium titanate ceramics (PZT) and polyvinylidene fluoride (PVDF). These wafers are thin, light, and unobstrusive and are very convenient for surface-bonded based inspection. Among these, however, PZT is usually preferred since PVDF has a high compliance and low inverse piezoelectric effect which results in poor actuator-sensor response. ${ }^{1}$

Several researchers have studied the guided wave field excited by different piezoceramic wafer transducers, and an extensive review of the field is presented in Ref. (1). Among them, Giurgiutiu ${ }^{2}$ considered the guided wave field excited by an infinitely wide PZT actuator bonded on the surface of an infinite isotropic plate, modeling the interaction between transducer and the plate through surface tractions at the transducer's edges. The proposed formulation was qualitatively validated through experimental results. Raghavan and Cesnik $^{3}$ used a similar approach to consider the excitation field produced by an arbitrarily shaped piezoelectric transducer. In that work, a formulation based on the 3-D equations of elasticity was used, and results were presented both for a general arbitrary shape and for the particular cases of rectangular, circular, and ring-shaped actuator configurations. Furthermore, expressions for the response of the wafers as sensors as well as experimental and finite element validations were presented.

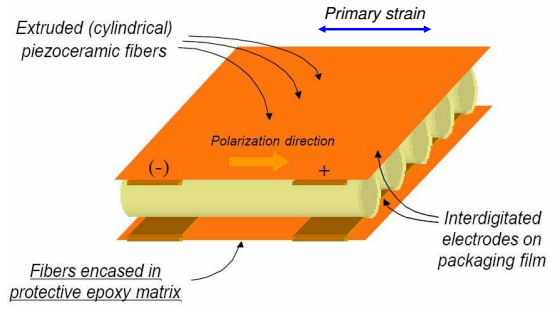

(a): AFC

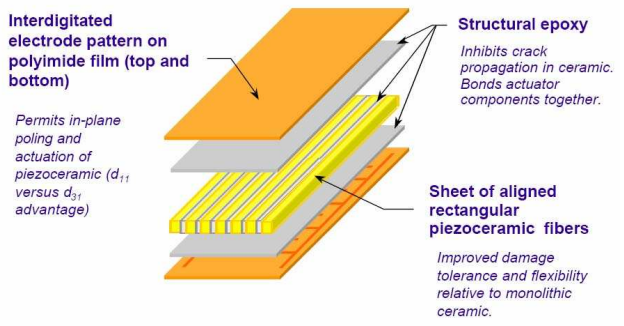

(b): MFC

Figure 1. The two primary types of APT. ${ }^{4}$

The main disadvantage in using piezoelectric wafers is that, although thin and light, the ceramic material is also very brittle and does not have good surface conformability. This limitation is particularly important for the shell-type structures usually encountered in aerospace applications. In order to alleviate this problem, different Anisotropic Piezocomposite Transducer (APT) concepts have been designed and manufactured in recent years. Bent and Hagood ${ }^{5}$ designed the Active Fiber Composite (AFC) transducer using extruded, cylindrical piezoceramic fibers embedded in an epoxy matrix. These fibers are actuated through the use of interdigitated electrode patterns printed on a copper-clad kapton film. This construction presented numerous advantages, in particular, a greatly enhanced surface conformability, high strain energy density, and focused strain actuation. The implementation of interdigitated electrodes allowed the use of the $3-3$ piezoelectric effect, where the poling of the device is such that the highest piezoelectric coupling coefficient coincides with the intended actuation direction, as shown in Fig. 1(a), allowing this device to induce strains at least twice as large as those obtained with simple PZT actuators. An alternative concept was developed by researchers at NASA Langley: the Macro-Fiber Composite (MFC) transducer, ${ }^{4}$ illustrated in Fig. 1(b). This type of construction is very similar to the AFC, except that it uses rectangular fibers obtained by dicing a piezoceramic wafer using high-precision dicing saws. ${ }^{6,7}$

The GW field excited by APT transducers has been recently modeled by Raghavan and Cesnik. ${ }^{8}$ In that work, the interaction between the transducer and the 3-D substrate was limited to shear forces along the transducer's edges in the direction of the piezoceramic fibers. Different structural configurations such as beams with rectangular cross-sections, cylinders, and infinite plates were considered. The results of that analysis showed the focused strain actuation that can be obtained with APT transducers. In addition, expressions for the sensor response of the MFC as well as experimental validation of the results were presented.

In a continuous effort towards focused GW generation, in the following sections, equations describing 
the GW field excited by a wedge-shaped APT will be derived and used in evaluating the performance of this geometry as actuator and sensor for large area SHM. First, the displacement equations corresponding to harmonic and time-dependent excitations will be derived, and the results compared to predictions from finite element (FE) simulations. Preliminary parametric studies are then carried out to find the best transducer dimensions and frequency of excitation for the proposed configuration. These studies will also discuss the focusing capability and directionality that can be obtained using this geometry. Finally, the response of APT and piezoelectric sensors under the GW field excited by the wedge-shaped APT actuator is derived, and parametric studies are conducted to identify optimal frequencies and dimensions.

\section{Boundary Value Problem Formulation}

The GW field excited by a finite-dimensional transducer bonded on the surface of an isotropic substrate is modeled as a boundary value problem of linear elasticity. The dynamics of the actuator and the substrate are assumed to be uncoupled, and their interaction is modeled as surface tractions along the edges of the transducer. There are two implications to this assumption. First, the bonding layer between the actuator and the substrate is assumed to be infinitely thin and not shear deformable, that is, the two parts are assumed to be perfectly bonded. This ensures that strains are transferred only along the actuator edges. This assumption is clearly an idealization, as the bonding layer will have a finite thickness in actual applications. Valuable insight into this situation was provided by the work of Crawley and de Luis, ${ }^{9}$ who considered the case of a piezoelectric actuator surface-bonded on a substrate under static conditions. They showed that, for a bonding layer with finite thickness, this assumption becomes accurate if the product of the actuator's modulus and thickness is much larger than that of the substrate on which it is bonded. In fact, it was shown that as this ratio approaches zero, the assumption becomes the exact solution. It was also shown that if this condition was not satisfied, a shear lag solution considering strain transfer along the length of the actuator was necessary. Secondly, so that the dynamics of the actuator can be ignored, its inertia must be a small fraction of the total inertia of the system in the region where strains are transferred. These assumptions are necessary if tractable semi-analytical solutions are to be obtained. Similar models have been used in the past and good agreement with experiments has been obtained. ${ }^{2,3,10,11}$

Based on these assumptions, consider an isotropic substrate of thickness $2 b$, with the coordinate system centered midway through its thickness, as depicted in Fig. 2(a). The substrate is of infinite dimensions along the $x$ - and $y$-directions, as shown in Fig. 2(b), and the transducer is bonded on the surface $z=b$. Using the constitutive law for isotropic materials and linear strain-displacement relations, the equilibrium equations in the absence of body forces may be expressed as:

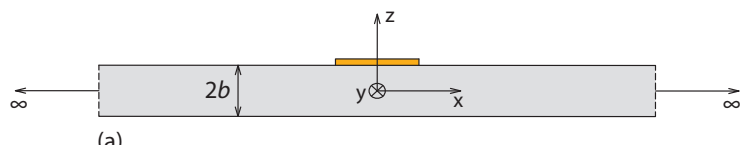

(a)

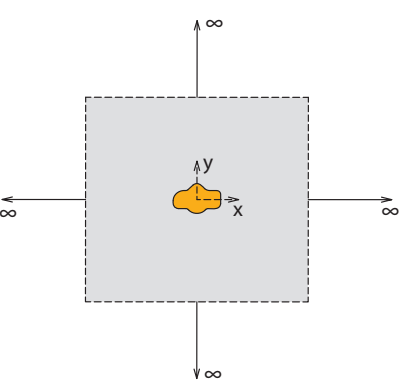

Figure 2. Transducer bonded on surface of infinite plate (a) Cross-sectional view (b) Top view.

$$
(\lambda+\mu) \nabla \nabla \mathbf{u}+\mu \nabla^{2} \mathbf{u}=\rho \ddot{\mathbf{u}}
$$

As previously mentioned, the transducer is replaced by surface tractions on the top surface of the substrate, while the bottom surface is traction free. Therefore, the following set of boundary conditions applies: 


$$
\begin{gathered}
\sigma(r, \theta,-b) \cdot \mathbf{n}_{\mathbf{l}}=\mathbf{0} \\
\sigma(r, \theta, b) \cdot \mathbf{n}_{\mathbf{u}}=\sigma_{0}
\end{gathered}
$$

where $\mathbf{n}_{\mathbf{l}}=\left[\begin{array}{lll}0 & 0 & -1\end{array}\right]^{T}, \mathbf{n}_{\mathbf{u}}=\left[\begin{array}{lll}0 & 0 & 1\end{array}\right]^{T}$ and:

$$
\sigma_{0}=\left[\begin{array}{c}
\sigma_{x z}^{0} \\
\sigma_{y z}^{0} \\
0
\end{array}\right]
$$

Equation (4) reflects the fact that the surface-bonded actuator only induces shear stresses on the substrate. A solution to this problem, for the general case of an arbitrarily-shaped transducer, was presented by Raghavan and Cesnik $^{3,10}$ and is outlined below. The displacement vector, $\mathbf{u}$, is decomposed into its Helmholtz components as:

$$
\begin{array}{r}
\mathbf{u}=\nabla \varphi+\nabla \times \mathbf{H} \\
\nabla \cdot \mathbf{H}=0
\end{array}
$$

which results in the following four differential equations:

$$
\begin{aligned}
\nabla^{2} \varphi & =\frac{\ddot{\varphi}}{c_{p}^{2}} \\
\nabla^{2} \mathbf{H} & =\frac{\ddot{\mathbf{H}}}{c_{s}^{2}}
\end{aligned}
$$

where $c_{p}$ and $c_{s}$ correspond to the dilatational and shear wave speeds, respectively, which are defined through:

$$
\begin{array}{r}
c_{p}^{2}=\frac{\lambda+2 \mu}{\rho} \\
c_{s}^{2}=\frac{\mu}{\rho}
\end{array}
$$

The solution to these equations, under harmonic excitation, was obtained using two-dimensional Fourier transforms, along with the set of boundary conditions given by Eqs. (2) and (3). The resulting displacement field can be separated into symmetric and antisymmetric components. To facilitate the presentation of the theory, only the results corresponding to the antisymmetric mode will be presented from this point on. The derivation of the symmetric component follows an analogous sequence. Note that the complete displacement field is obtained by summing the contribution from both modes. Thus, the cartesian displacement components, expressed in cartesian coordinates, were expressed as:

$$
\mathbf{u}_{A}(x, y, t)=\int_{-\infty}^{\infty} \int_{-\infty}^{\infty} \frac{\boldsymbol{\Gamma}_{A}\left(\xi_{x}, \xi_{y}\right)}{D_{A}(\xi)} \boldsymbol{\Psi}_{A}\left(\xi_{x}, \xi_{y}\right) \overline{\mathbf{F}}\left(\xi_{x}, \xi_{y}\right) e^{-i\left(\xi_{x} x+\xi_{y} y-\omega t\right)} d \xi_{x} d \xi_{y}
$$

Note that Eq. (8) provides the displacement components at the surface $z=b$. All subsequent analytical expressions presented here will be for this surface as well. In Eq. (8), $\boldsymbol{\Gamma}_{A}$ and $\boldsymbol{\Psi}_{A}$ are matrices of coefficients, $\overline{\mathbf{F}}$ is a vector containing the Fourier transform of the surface tractions, and $D_{A}$ corresponds to the dispersion relation for the antisymmetric mode of Rayleigh-Lamb waves, given by:

$$
D_{A}=\left(\xi^{2}-\beta^{2}\right)^{2} \sin \alpha b \cos \beta b+4 \xi^{2} \alpha \beta \cos \alpha b \sin \beta b
$$

where $\xi^{2}=\xi_{x}^{2}+\xi_{y}^{2}$. The terms $\alpha$ and $\beta$ are defined below: 


$$
\begin{aligned}
& \alpha \equiv \sqrt{\frac{\omega^{2}}{c_{p}^{2}}-\xi^{2}} \\
& \beta \equiv \sqrt{\frac{\omega^{2}}{c_{s}^{2}}-\xi^{2}}
\end{aligned}
$$

In the following section, the result given by Eq. (8) will be expressed in polar coordinates, and used to solve for the displacement field produced by a wedge-shaped APT.

\section{Wedge-shaped APT}

The wedge-shape geometry is easily described using polar coordinates, and is defined by the transducer's inner and outer radii, $R_{I}$ and $R_{O}$, as well as its left- and right-most angular edges, $\theta_{L}$ and $\theta_{R}$. Figure 3(a) shows a schematic of the transducer's geometry. At this point, Eq. (8) must be modified to express the displacement components in polar coordinates. This modification involves the use of the 2-D Fourier transform for polar coordinates; for a generic function, $g(r, \theta)$, it is defined as:

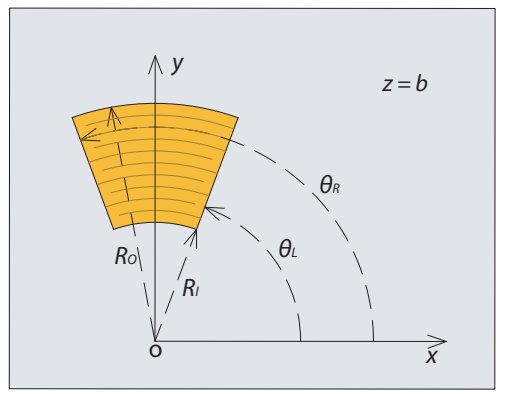

(a)

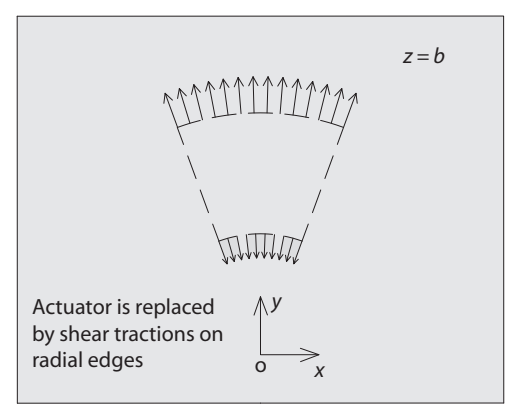

(b)

Figure 3. (a) Geometry of wedge-shaped APT; (b) Transducer replaced by shear tractions along radial edges.

$$
G(\xi, \phi)=\int_{0}^{\infty} \int_{0}^{2 \pi} g(r, \theta) e^{i \xi r \cos (\theta-\phi)} r d \theta d r
$$

while the inverse transform is defined through:

$$
g(r, \theta)=\frac{1}{4 \pi^{2}} \int_{0}^{\infty} \int_{0}^{2 \pi} G(\xi, \phi) e^{-i \xi r \cos (\theta-\phi)} \xi d \phi d \xi
$$

Using these definitions, along with the transformations $\xi_{x}=\xi \cos \phi$ and $\xi_{y}=\xi \sin \phi$, yields the following equation for the cartesian displacement components expressed in polar coordinates:

$$
\mathbf{u}_{A}(r, \theta, t)=\int_{0}^{2 \pi} \int_{0}^{\infty} \frac{\boldsymbol{\Gamma}_{A}(\xi)}{D_{A}(\xi)} \boldsymbol{\Psi}_{A}(\xi, \phi) \overline{\mathbf{F}}(\xi, \phi) e^{-i \xi r \cos (\theta-\phi)} e^{i \omega t} d \xi d \phi
$$

The matrix $\boldsymbol{\Gamma}_{A}$ is a $3 \times 3$ diagonal matrix of coefficients whose components are given by:

$$
\begin{array}{r}
\Gamma_{11}=\Gamma_{22}=\frac{\tau_{0} \sin \beta b \xi}{4 \pi^{2} \mu \beta \cos \beta b} \\
\Gamma_{33}=\frac{-i \tau_{0} \xi^{2}}{4 \pi^{2} \mu}\left[2 \alpha \beta \cos \alpha b \sin \beta b+\left(\xi^{2}-\beta^{2}\right) \cos \beta b \sin \alpha b\right]
\end{array}
$$

Similarly, $\boldsymbol{\Psi}_{A}$ is a $3 \times 2$ matrix of coefficients defined through: 


$$
\boldsymbol{\Psi}_{A}=\left[\begin{array}{cc}
-\gamma_{3}^{(1)}-\gamma_{4}^{(1)}\left(e^{-2 i \phi}+e^{2 i \phi}\right) & \gamma_{5}^{(1)}\left(e^{2 i \phi}-e^{-2 i \phi}\right) \\
\gamma_{1}^{(2)}\left(e^{2 i \phi}-e^{-2 i \phi}\right) & -\gamma_{4}^{(2)}-\gamma_{5}^{(2)}\left(e^{-2 i \phi}+e^{2 i \phi}\right) \\
\cos \phi & \sin \phi
\end{array}\right]
$$

where the coefficients $\gamma_{i}^{(j)}$ (defined in the Appendix) depend on the substrate material properties, frequency, and wavenumbers. Finally, the vector $\overline{\mathbf{F}}$ contains the Fourier transform of the shear tractions produced at the transducer's edges. Only shear stresses along the piezoceramic fiber direction are considered, as shown in Fig. 3(b). This choice is based on the fact that the transducer is acting on the $3-3$ piezoelectric effect, which makes the strains induced along the fiber's axis significantly larger than those along its normal direction. For a typical piezoelectric material (such as PZT-5A) poled along the thickness direction, the piezoelectric coupling coefficient normal to the poling direction is approximately $54 \%$ smaller than that along the poling direction. ${ }^{12}$ This level of actuation is still significant, but the strains induced along this direction are further attenuated due to the high aspect ratio of the fiber and the construction of the APT device. As previously discussed, the APT is a composite transducer with epoxy located in between any two fibers. Thus, the shear lag effect effectively eliminates any strain transmitted normal to the fiber length. As a result, for the case of a wedge-shaped APT, the boundary conditions given by Eq. (3) take the form:

$$
\begin{gathered}
\sigma_{z r}(r, \theta, b)=\tau_{0} f(r, \theta) \\
\sigma_{z z}(r, \theta, b)=\sigma_{z \theta}(r, \theta, b)=0
\end{gathered}
$$

where $\tau_{0}$ represents the force per unit length exerted by the transducer on the substrate, and $f(r, \theta)$ is a function whose purpose is to make the stress non-zero only along the transducer's radial edges, as shown in Fig. 3(b). Such an expression is given by ${ }^{a}$ :

$$
f(r, \theta)=\left[u\left(\theta-\theta_{L}\right)-u\left(\theta-\theta_{R}\right)\right]\left[\delta\left(r-R_{I}\right)-\delta\left(r-R_{O}\right)\right]
$$

In order to use the formulation presented above, the Fourier transform of Eq. (19) must be determined. Before doing so, the function must be decomposed along the $x$ - and $y$-directions to be compatible with the displacement vector given by Eq. (14). This is simply done by defining the $x$ - and $y$-components as the function multiplied by $\cos \theta$ and $\sin \theta$, respectively. Then, the necessary Fourier transforms are given by:

$$
\begin{aligned}
& \overline{F_{1}}(\xi, \phi)=\int_{0}^{2 \pi} \int_{0}^{\infty}\left[u\left(\theta-\theta_{L}\right)-u\left(\theta-\theta_{R}\right)\right]\left[\delta\left(r-R_{I}\right)-\delta\left(r-R_{O}\right)\right] \cos \theta e^{i \xi r \cos (\theta-\phi)} r d r d \theta \\
& \overline{F_{2}}(\xi, \phi)=\int_{0}^{2 \pi} \int_{0}^{\infty}\left[u\left(\theta-\theta_{L}\right)-u\left(\theta-\theta_{R}\right)\right]\left[\delta\left(r-R_{I}\right)-\delta\left(r-R_{O}\right)\right] \sin \theta e^{i \xi r \cos (\theta-\phi)} r d r d \theta
\end{aligned}
$$

The integrals given by Eqs. (20) and (21) cannot be solved analytically. An alternate solution method is applicable since the radial and angular parts of the function are readily separable. For this type of functions, the Hankel transform of the radial part can be combined with a Fourier series representation of the angular part to obtain the desired Fourier transform. ${ }^{13}$ In this way, the two-dimensional Fourier transform, $W(\xi, \phi)$, for a generic function $w(r, \theta)=g(\theta) h(r)$ can be expressed as:

$$
W(\xi, \phi)=\sum_{k=-\infty}^{\infty} c_{k} e^{i k \phi}(-i)^{k} \widetilde{H}_{k}(\xi)
$$

where $\widetilde{H}_{k}$ represents the $k^{t h}$ order Hankel transform of $h(r)$, defined through:

$$
\widetilde{H}_{k}(\xi)=2 \pi \int_{0}^{\infty} h(r) J_{k}(r \xi) r d r
$$

and $c_{k}$ are the complex Fourier coefficients of the function $g(\theta)$, defined by:

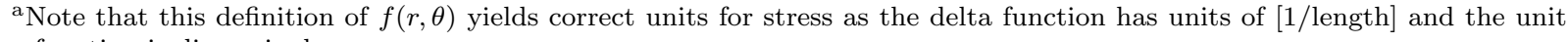
step function is dimensionless.
} 


$$
c_{k}=\frac{1}{2 \pi} \int_{0}^{2 \pi} g(\theta) e^{-i k \theta} d \theta
$$

In the case under consideration, the function $h(r)$ is defined by:

$$
h(r)=\delta\left(r-R_{I}\right)-\delta\left(r-R_{O}\right)
$$

while the two functions $g_{1}(\theta)$ and $g_{2}(\theta)$ are given by:

$$
\begin{aligned}
& g_{1}(\theta)=\left[u\left(\theta-\theta_{L}\right)-u\left(\theta-\theta_{R}\right)\right] \cos (\theta) \\
& g_{2}(\theta)=\left[u\left(\theta-\theta_{L}\right)-u\left(\theta-\theta_{R}\right)\right] \sin (\theta)
\end{aligned}
$$

Therefore, the necessary Fourier transforms for the shear tractions applied on the substrate's surface result in:

$$
\overline{F_{j 1}}=\sum_{k=-\infty}^{\infty} c_{k}^{(j)} e^{i k \phi}(-i)^{k} \chi_{k}, \quad j=1,2
$$

where:

$$
\chi_{k}=2 \pi\left[R_{O} J_{k}\left(\xi R_{O}\right)-R_{I} J_{k}\left(\xi R_{I}\right)\right]
$$

The resulting complex Fourier coefficients, obtained by substituting Eqs. (26) into Eq. (24), can be expressed through:

$$
\begin{gathered}
c_{k}^{(1)}=\frac{u\left(\theta_{L}\right)\left[e^{-i k \theta_{L}}\left(i k \cos \theta_{L}-\sin \theta_{L}\right)-i k\right]-u\left(\theta_{R}\right)\left[e^{-i k \theta_{R}}\left(i k \cos \theta_{R}-\sin \theta_{R}\right)-i k\right]}{2 \pi\left(1-k^{2}\right)}, \quad|k| \neq 1 \\
c_{k}^{(1)}=\frac{\left(2 \pi-\theta_{L}-\sin \theta_{L} e^{-i \theta_{L}}\right) u\left(\theta_{L}\right)-\left(2 \pi-\theta_{R}-\sin \theta_{R} e^{-i \theta_{R}}\right) u\left(\theta_{R}\right)}{4 \pi}, \quad|k|=1 \\
c_{k}^{(2)}=\frac{u\left(\theta_{L}\right)\left[e^{-i k \theta_{L}}\left(\cos \theta_{L}+i k \sin \theta_{L}\right)-1\right]-u\left(\theta_{R}\right)\left[e^{-i k \theta_{R}}\left(\cos \theta_{R}+i k \sin \theta_{R}\right)-1\right]}{2 \pi\left(1-k^{2}\right)},|k| \neq 1 \\
c_{k}^{(2)}=\frac{u\left(\theta_{L}\right)\left(-1+e^{-2 i \theta_{L}}-4 i \pi+2 i \theta_{L}\right)-u\left(\theta_{R}\right)\left(-1+e^{-2 i \theta_{R}}-4 i \pi+2 i \theta_{R}\right)}{8 \pi},|k|=1
\end{gathered}
$$

\section{A. Solution Process: Region III}

To facilitate the solution process, the domain is subdivided into three regions, as depicted in Fig. 4. Region III is defined as the set of all points such that $r>R_{O}$. This set is characterized by the fact that both edges of the transducer, inner and outer, send waves propagating in the positive radial direction (henceforth referred to as outward direction). The form of the solution, i.e., the combination of Eqs. (14) and (27), suggests that the integral definition of the Bessel function of $k^{t h}$ order be used:

$$
J_{k}(z)=\frac{1}{2 \pi} \int_{0}^{2 \pi} e^{-i \frac{\pi}{2} k} e^{i z \cos \phi} e^{i k \phi} d \phi=\frac{1}{2 \pi} \int_{0}^{2 \pi} e^{i \frac{\pi}{2} k} e^{-i z \cos \phi} e^{-i k \phi} d \phi
$$

Note that Eq. (31) holds because the Bessel function returns a real number for $z>0$. Therefore, taking the complex conjugate of the integrand does not alter the final result as its imaginary part is zero. This equation must be manipulated so that the exponential part of its integrand is similar to that in Eq. (14). This process is carried out through the following change of variables:

$$
\phi=\bar{\phi}-\theta
$$

This operation results in: 


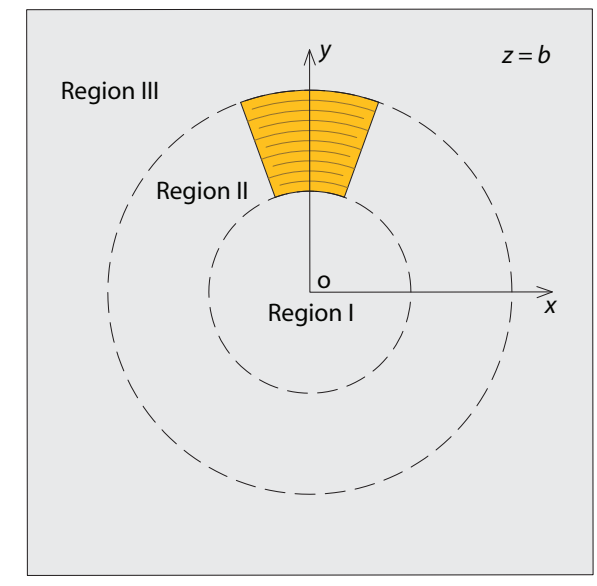

Figure 4. Domain is subdivided into three separate regions according to wave behavior.

$$
J_{k}(z)=\frac{1}{2 \pi} \int_{\theta}^{2 \pi+\theta} \zeta_{k} e^{-i z \cos (\theta-\bar{\phi})} e^{-i k \bar{\phi}} d \bar{\phi}
$$

where:

$$
\zeta_{k} \equiv e^{i \frac{\pi}{2} k} e^{i k \theta}
$$

The right hand side in Eq. (33) is almost in the desired form. The only differences between this result and the form of Eq. (14) are in the integration limits and the index $k$ in the complex exponential. The difference in the integration limits is of no concern since the integrand of both functions is periodic in $\phi$ with a period of $2 \pi$. The indices in Eq. (14) will vary according to the powers of the exponentials given in Eq. (16). This can be simply resolved by redefining the resulting index of the complex exponential as $-k$, and incorporating this change in the corresponding multiplying coefficients. As a result, the solution to Eq. (14) in the angular wavenumber domain is given by:

$$
\mathbf{u}_{A}(r, \theta, t)=\int_{0}^{\infty} \sum_{k=-\infty}^{\infty}\left[\frac{2 \pi \boldsymbol{\Gamma}_{A}(\xi)}{\zeta_{k}(\theta) D_{A}(\xi)} \boldsymbol{\Delta}_{A}(k, \xi) J_{k}(\xi r)\right] e^{i \omega t} d \xi
$$

where $\boldsymbol{\Delta}_{A}$ is a $3 \times 1$ column vector of coefficients defined in the Appendix. The Bessel function solution presented in Eq. (35) corresponds to a standing wave. In order to obtain a propagating wave, we resort to the following definition of the Hankel function of the first and second kind:

$$
\begin{aligned}
& H_{k}^{(1)}(z)=J_{k}(z)+i Y_{k}(z) \\
& H_{k}^{(2)}(z)=J_{k}(z)-i Y_{k}(z)
\end{aligned}
$$

Based on the frequency convention we have adopted, the Hankel function of the second kind corresponds to an outward-propagating wave in time. Therefore only this part is retained, which yields:

$$
\mathbf{u}_{A}(r, \theta, t)=\int_{-\infty}^{\infty} \sum_{k=-\infty}^{\infty}\left[\frac{\pi \boldsymbol{\Gamma}_{A}(\xi)}{\zeta_{k}(\theta) D_{A}(\xi)} \boldsymbol{\Delta}_{A}(k, \xi) H_{k}^{(2)}(\xi r)\right] e^{i \omega t} d \xi
$$

Note that the integration limits in the radial wavenumber domain have changed. This is due to the fact that retaining the Hankel function of the second kind only is equivalent to replacing the azimuthal wavenumber integration limits from a range of $2 \pi$ to a range of $\pi$; hence, in order to keep the integration domain unchanged, the limits in the radial wavenumber domain must be modified ${ }^{\mathrm{b}}$. The resulting integral

\footnotetext{
${ }^{\mathrm{b}}$ This statement is not mathematically rigorous as a correction term included in the integral definition of $H_{k}^{(2)}$ has been neglected. However, it can be shown that the contribution from this term is only significant for small arguments.
} 
is solved using the residue theorem from complex calculus. Since Eq. (35) is a rational function of $\xi$, it follows from the theory of complex calculus that the residue, $\bar{b}$, of this function at a pole $\xi_{A}$ can be expressed as: ${ }^{14}$

$$
\bar{b}=\frac{N_{A}\left(\xi_{A}\right)}{D_{A}^{\prime}\left(\xi_{A}\right)}
$$

where the 'symbol indicates differentiation with respect to $\xi$, and the pole $\xi_{A}$ corresponds to values of $\xi$ for which $D_{A}$ vanishes; these points are the wavenumbers corresponding to the antisymmetric mode of Rayleigh-Lamb waves at a frequency $\omega$. The notation $N(\xi)$ has been used in Eq. (38) to illustrate the concept. It is important to note that Eq. (38) holds as long as $N\left(\xi_{A}\right)$ does not equal zero. This result can be combined with the residue theorem to express the solution of the integral in the $\xi$ domain as:

$$
\int_{-\infty}^{\infty} \frac{N(\xi)}{D(\xi)} d \xi=2 \pi i \sum_{\xi_{A}} \frac{N\left(\xi_{A}\right)}{D^{\prime}\left(\xi_{A}\right)}, \quad \xi_{A}>0
$$

where the condition that $\xi_{A}$ be greater than zero indicates that only positive wavenumbers are to be included in the integration contour. Therefore, the solution may be expressed in final form as:

$$
\mathbf{u}_{A}(r, \theta, t)=\sum_{k=-\infty}^{\infty}\left[\frac{2 \pi^{2} i \boldsymbol{\Gamma}_{A}\left(\xi_{A}\right)}{\zeta_{k}(\theta) D_{A}^{\prime}\left(\xi_{A}\right)} \boldsymbol{\Delta}_{A}\left(k, \xi_{A}\right) H_{k}^{(2)}\left(\xi_{A} r\right)\right] e^{i \omega t}
$$

Note that since only harmonic excitation is being considered, only one wavenumber needs to be included. The solution given by Eq. (40) corresponds to the antisymmetric mode. The symmetric mode is given by a similar equation, which is derived by interchanging all sine and cosine terms whose arguments depend on the substrate half-thickness $b$. This is a crucial change since it modifies the dispersion equation $D_{A}$, which produces solutions with different wavenumbers.

\section{B. Solution Process: Region I}

In a manner analogous to the one presented in section A, region I is defined as the set of all points such that $r<R_{I}$. This set is characterized by the fact that both edges of the transducer send waves in the negative radial direction (henceforth referred to as inward direction). In this case, the solution is obtained as an infinite sum of standing waves. Note that each term in the sum represents a standing wave, but the overall combination yields a propagating wave. The expression for the antisymmetric displacement components induced under harmonic excitation at a frequency $\omega$ is given by:

$$
\mathbf{u}_{A}=\sum_{k=-\infty}^{\infty} \frac{2 i \pi^{2} \boldsymbol{\Gamma}_{A}\left(\xi_{A}\right)}{\zeta_{k}(\theta) D_{A}^{\prime}\left(\xi_{A}\right)} \boldsymbol{\Lambda}_{A}\left(k, \xi_{A}\right) J_{k}\left(\xi_{A} r\right) e^{i \omega t}
$$

where $\boldsymbol{\Lambda}_{A}$ is a $3 \times 1$ column vector of coefficients, presented in the Appendix.

\section{Solution Process: Region II}

Region II is defined as the set of all points such that $R_{I}<r<R_{O}$. It is characterized by the fact that the outer edge of the transducer produces inward-propagating waves, while its inner edge produces outwardpropagating waves. The solution for this region is obtained as a combination of the solutions presented in sections A and B. The contribution from the outer radius is expressed as a sum of standing waves, while that from the inner radius is expressed through Hankel functions of the second kind. The antisymmetric displacement components under harmonic excitation at a frequency $\omega$ are given by:

$$
\mathbf{u}_{A}=\sum_{k=-\infty}^{\infty} \frac{2 i \pi^{2} \boldsymbol{\Gamma}_{A}\left(\xi_{A}\right)}{\zeta_{k}(\theta) D_{A}^{\prime}\left(\xi_{A}\right)}\left[\bar{\Upsilon}_{A}\left(k, \xi_{A}\right) J_{k}\left(\xi_{A} r\right)-\boldsymbol{\Upsilon}_{A}\left(k, \xi_{A}\right) H_{k}^{(2)}\left(\xi_{A} r\right)\right] e^{i \omega t}
$$

where $\boldsymbol{\Upsilon}_{A}$ and $\bar{\Upsilon}_{A}$ are vectors of coefficients whose definition is given in the Appendix. 


\section{Toneburst Response}

In typical SHM applications, the structure to be inspected for damage is excited with a stress wave whose shape is determined by a time-dependent modulated signal. This type of signal is generally desired so as to control the frequency bandwidth and avoid dispersion. Hann-modulated signals have been successfully used in the past by several researchers and will be adopted in the present formulation. ${ }^{15}$ Such a signal is given by:

$$
s(t)=\frac{1}{2}\left[1-\cos \left(2 \frac{\omega t}{n}\right)\right] \sin \left(\omega_{0} t\right)
$$

where $n$ is the number of half-cycles and $\omega_{0}$ represents the center frequency of excitation. In order to account for the time-dependence, the time Fourier transform of Eq. (43) must be determined through:

$$
S(\omega)=\int_{-\infty}^{\infty} s(t) e^{-i \omega t} d t
$$

Substituting Eq. (43) into Eq. (44) yields the following expression for the time Fourier transform of the toneburst signal:

$$
S(\omega)=\frac{-3 n^{2} \omega^{2} \omega_{0}^{2}+4 \omega_{0}^{3}-n^{2} \omega_{0}^{3}+\left[n^{2}\left(\omega^{2}+3 \omega_{0}^{2}\right)-4 \omega_{0}^{2}\right]\left[e^{\frac{i n \omega \pi}{\omega_{0}}} \omega_{0} \cos n \pi-i e^{\frac{i n \omega \pi}{\omega_{0}}} \omega \sin n \pi\right]}{\left(\omega_{0}^{2}-\omega^{2}\right)\left[16 \omega_{0}^{4}+n^{4}\left(\omega^{2}-\omega_{0}^{2}\right)^{2}-8 n^{2} \omega_{0}^{2}\left(\omega^{2}+\omega_{0}^{2}\right)\right]}
$$

For the particular case when $\omega$ and $\omega_{0}$ are equal, the following expression applies:

$$
S(\omega)=\frac{1-e^{2 i n \pi}+2 i n \pi\left(1-n^{2}\right)}{8 \omega_{0}\left(1-n^{2}\right)}
$$

The time-dependent displacements are obtained through the inverse Fourier transform of the product of the transforms of the spatial and temporal parts. Since the excitation signal has several frequency components, a sum over all possible wavenumbers is necessary. Hence, the solution becomes (only antisymmetric mode in region III is presented for simplicity):

$$
\mathbf{u}_{A}(r, \theta, t)=\frac{1}{2 \pi} \int_{-\infty}^{\infty} \sum_{\xi_{A}}\left[\sum_{k=-\infty}^{\infty} \frac{i \pi^{2} \boldsymbol{\Gamma}_{A}\left(\xi_{A}\right)}{\zeta_{k}(\theta) D_{A}^{\prime}\left(\xi_{A}\right)} \boldsymbol{\Delta}_{A}\left(k, \xi_{A}\right) H_{k}^{(2)}\left(\xi_{A} r\right)\right] S(\omega) e^{i \omega t} d \omega
$$

\section{Finite Element Validation}

Results from the analytical formulation presented above were compared with three-dimensional FE simulations run in ABAQUS ${ }^{16}$ to assess its spatial and temporal performance. Taking advantage of the problem's symmetries, only one eighth of an aluminum plate was considered. A summary of the material properties and actuator dimensions considered in the simulations is given in Table 1.

Table 1. Substrate material properties and actuator dimensions used in FE simulations and analytical results.

\begin{tabular}{lc} 
Parameter & Value \\
\hline $\mathrm{E},[\mathrm{GPa}]$ & 70 \\
$\nu$ & 0.33 \\
$\rho,\left[\mathrm{kg} / \mathrm{m}^{3}\right]$ & 2700 \\
$R_{O},[\mathrm{~m}]$ & 0.015 \\
$R_{I},[\mathrm{~m}]$ & 0.005 \\
$\Delta \theta_{a},[\mathrm{deg}]$ & 30
\end{tabular}

The mesh consisted of three-dimensional continuum elements, and was primarily composed of eight-node bricks (C3D8). In addition, the geometry of the mesh required the use of six-node tetrahedra (C3D6) for the elements immediately connected to the origin. Similarly, infinite continuum elements (CIN3D8) were used on 
the outer radial boundary of the model, in an effort to minimize boundary reflections. ${ }^{16}$ However, the results showed that these elements were not successful in achieving this, and therefore the radial positions selected for comparison were located far from the radial edge. In all cases, three elements were used through the thickness of the plate. A schematic of the mesh is shown in Fig. 5(a), while its relevant dimensions (radius, $R$, and half-thickness, $b)$ are given in Table 2 . Symmetric $\left(\mathrm{S}_{0}\right)$ and antisymmetric $\left(\mathrm{A}_{0}\right)$ modes were excited by specifying a symmetry and antisymmetry condition with respect to the $z$-axis, respectively. The symmetric mode was used to validate the out-of-plane displacement in region III, while the antisymmetric mode was used to model the in-plane displacements. This choice was based on the fact that the antisymmetric mode has a higher frequency threshold for the appearance of the SH-mode (shear horizontal mode present only in the in-plane displacements), which is not considered in the analytical solution and would therefore prevent an accurate validation.

The radial dimension of the elements, $\Delta r$, was selected so as to have at least 20 nodes per wavelength for the highest frequency of the toneburst excitation. The azimuthal size, $\Delta \theta$, was selected at three degrees providing six nodes along the angular span of the actuator, whose centerline was located at 90 degrees. A summary of the mesh parameters is presented in Table 2. The shear tractions caused by the actuator were modeled through nodal forces on the nodes corresponding to the actuator edges. Since the analytical model considers the force per unit length to be constant along the radial edges of the transducer, the relative force amplitudes must be scaled appropriately. This was achieved by considering point forces of unit magnitude on the outer radial edge and scaling the magnitude on the inner edge accordingly, which results in its amplitude being $R_{I} / R_{O}$. The time-dependent part of this forcing function was a Hann-modulated toneburst whose properties are also summarized in Table 2. A schematic showing the details of the shear traction application is given in Fig. 5(b). The time step, $\Delta t$, was selected so as to satisfy the following criteria: (i) proper sampling of the highest frequency component of the excitation signal, and (ii) sufficient resolution of the time needed for the fastest traveling wave to move across one element in the radial direction. ${ }^{17}$ An implicit dynamic analysis was performed with 1404 steps in all cases.

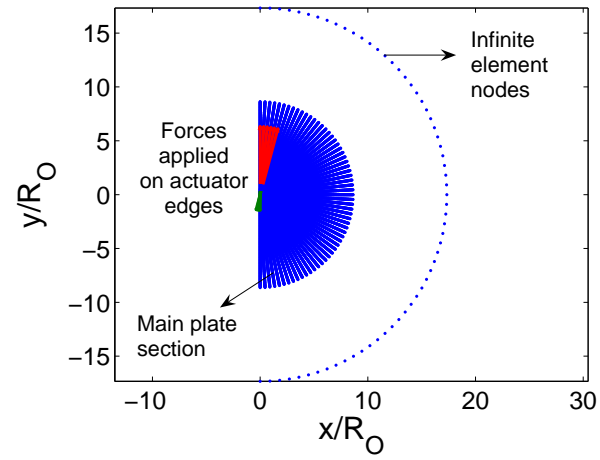

(a)

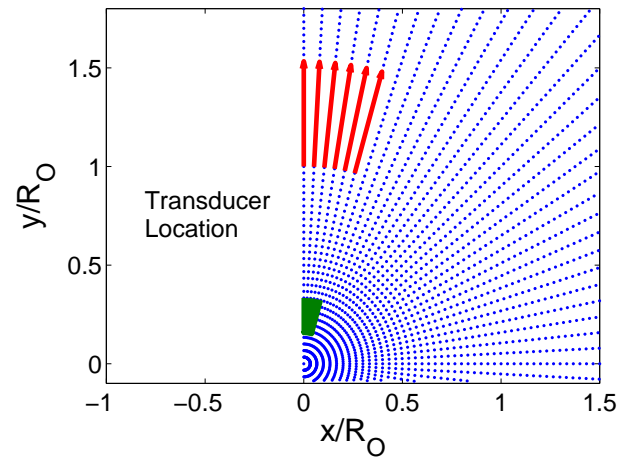

(b)

Figure 5. FE mesh: (a) Complete overview; (b) Detail on shear traction application points.

The numerical implementation of the analytical solution requires that a finite number of terms in the infinite sum given by Eq. (47) be selected. This number was chosen based on the normalized error between the angular part of the shear traction function, Eq. (26), and its Fourier series representation, Eq. (22). This error is defined as:

$$
\varepsilon=\frac{\sqrt{\int_{0}^{2 \pi}\left|g_{1,2}-g_{1,2}^{F}\right|^{2} d \theta}}{\sqrt{\int_{0}^{2 \pi} g_{1,2}^{2} d \theta}}
$$

where the superscript $F$ refers to the Fourier series representation of the function. Figure 6 shows how this error decreases with increasing number of terms. Based on this result, the sum was truncated at 150 terms as the reduction in error with increasing terms was slower after this point. For the spatial validation, several azimuthal positions were selected at different radial locations sufficiently far from the radial edge to avoid 
Table 2. Mesh and analysis parameters used in FE simulations.

\begin{tabular}{lcc} 
Parameter & Value $\left(\mathrm{S}_{0}\right)$ & Value $\left(\mathrm{A}_{0}\right)$ \\
\hline$R,[\mathrm{~m}]$ & 0.54 & 0.13 \\
$\Delta r,[\mathrm{~m}]$ & 0.0025 & 0.0005 \\
$\Delta \theta,[\mathrm{deg}]$ & 3 & 3 \\
$\Delta t,[\mathrm{~s}]$ & $10^{-7}$ & $10^{-7}$ \\
$b,[\mathrm{~m}]$ & 0.002 & 0.002 \\
$f_{0},[\mathrm{kHz}]$ & 100 & 100 \\
$n$ & 7 & 7 \\
No. of Elements & 38700 & 47336 \\
No. of Nodes & 52464 & 63444
\end{tabular}

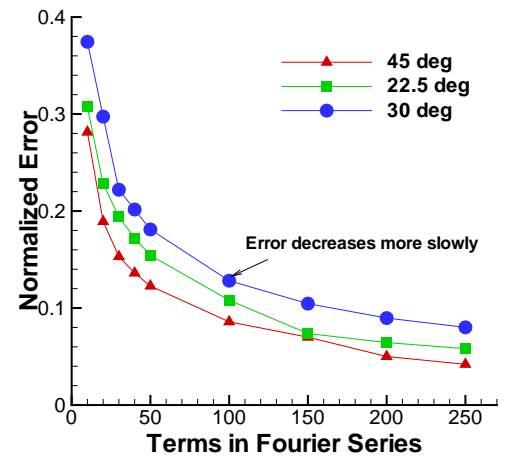

(a)

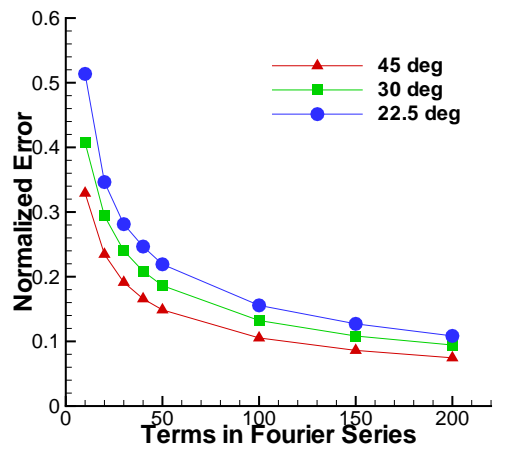

(b)

Figure 6. Normalized error between angular part of shear traction function and its Fourier series representation for: (a) $x_{1}$-component; (b) $x_{2}$-component.

boundary reflections. Figure 7(a) shows the set of points selected for comparisons in region III. The radial positions selected were ten times the transducer's outer radius $(\hat{n}=10)$ for the symmetric mode, and five thirds times the outer radius $(\hat{n}=5 / 3)$ for the antisymmetric mode. Seven azimuthal locations from the transducer's centerline to its opposite direction were selected, which resulted in intervals of 30 degrees. Figure 7(b) shows the normalized peak-to-peak comparison between analytical and FE results, while Figs. 7(c)-(d) show a similar result for the in-plane displacements. These figures indicate that the spatial distribution is accurately modeled by the analytical solution.

A similar comparison was performed for regions I and II, where only the antisymmetric mode was considered due to its shorter wavelength. Figure 8(a) shows a schematic of the points selected for comparison. For region I, an azimuthal distribution similar to the one used in the previous case was employed, but the radial position was changed to one-half the transducer's inner radius. In region II, only six azimuthal points were considered to avoid having a point over the transducer's area, while the radial location selected was the transducer's midpoint along the radial direction. Figure 8(b)-(c) show good spatial correlation for the out-of-plane displacement in regions I and II, respectively. Similarly, Fig. 8(d) shows that the time history of the analytical displacements also matches very well with $\mathrm{FE}$ results. 


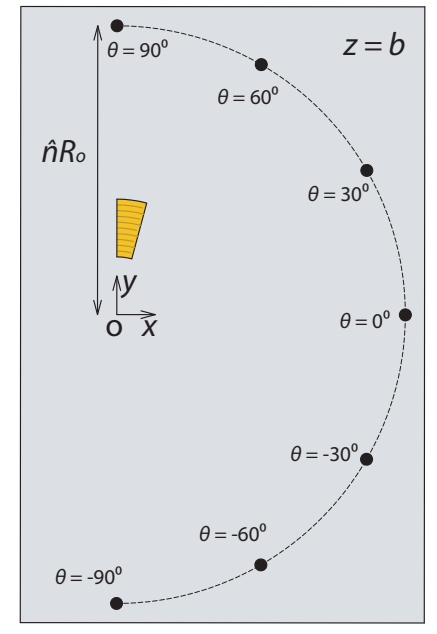

(a)

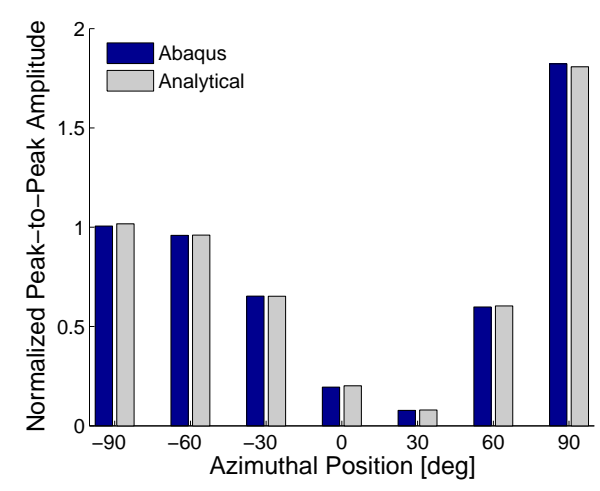

(c)

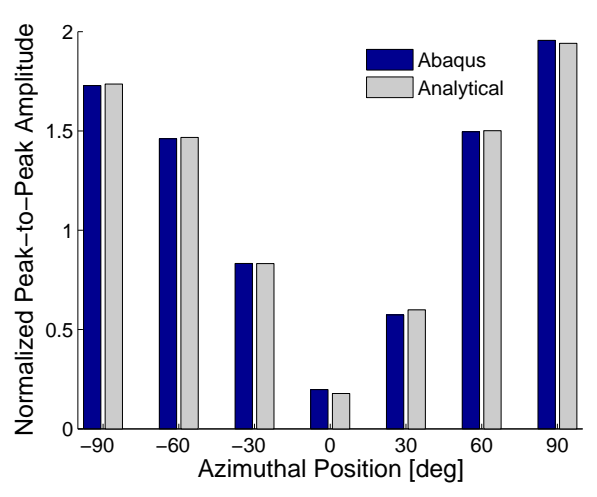

(b)

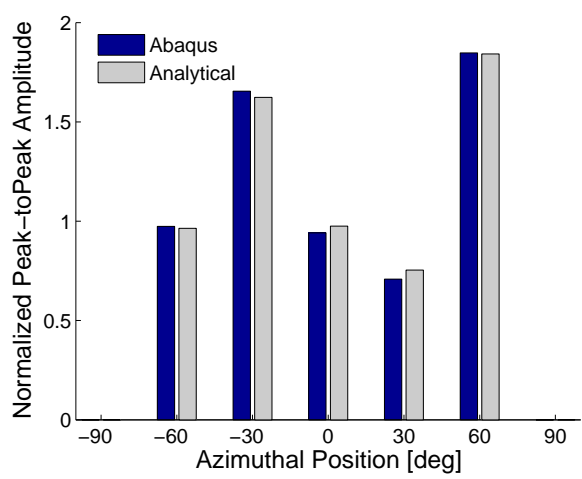

(d)

Figure 7. Comparison between FE and analytical results in region III (a) Points selected for comparison (b) Amplitude comparison for symmetric $u_{3}$ (c) Amplitude comparison for antisymmetric $u_{2}$ (d) Amplitude comparison for antisymmetric $u_{1}$.

\section{Parametric Studies}

\section{A. Actuator Dimensions}

The objective of these studies was to determine how the transducer dimensions, namely its radial length and azimuthal span, influenced the induced displacement field over a wide frequency range. The reference dimension chosen for the variations in the radial dimension was the substrate half-thickness, $b$, as it is the only length parameter that affects dispersion. This resulted in the definition of the non-dimensional parameter $\Delta r_{a} / b$ which was varied to compute the results presented here. The metric chosen in this case was the out-of-plane displacement.

The results for various azimuthal spans are shown in Figs. 9 and 10 for the symmetric and antisymmetric modes, respectively. The transducer centerline was located at $\theta=90^{\circ}$ and the radial position selected for comparison was $15 R_{O}$. The substrate thickness was kept constant at $b=0.001 \mathrm{~m}$, while variations in the non-dimensional parameter were obtained by fixing the transducer's outer radius $\left(R_{O}=0.025 \mathrm{~m}\right)$ and varying the inner radius so as to achieve the desired ratio.

The results have been normalized by the maximum amplitude in each set, that is, only quantitative comparisons among different transducer radial sizes are presented. A distinction between the two modes is not considered because it is known that the out-of-plane displacement corresponding to the $\mathrm{A}_{0}$ mode is significantly stronger than that for the $\mathrm{S}_{0}$ mode. ${ }^{15}$ Thus, a quantitative comparison between both modes 


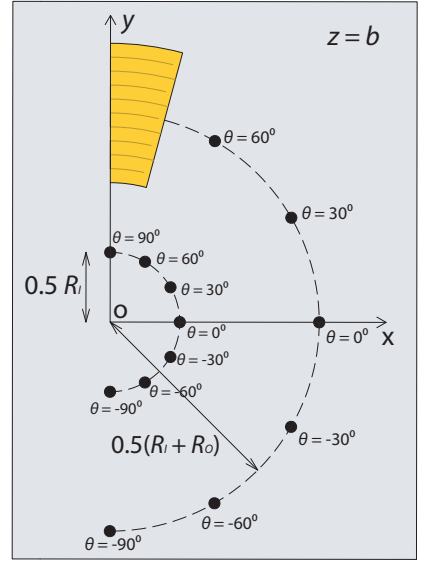

(a)

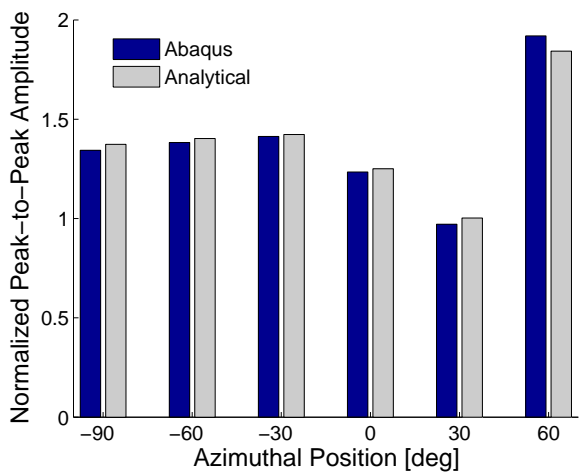

(c)

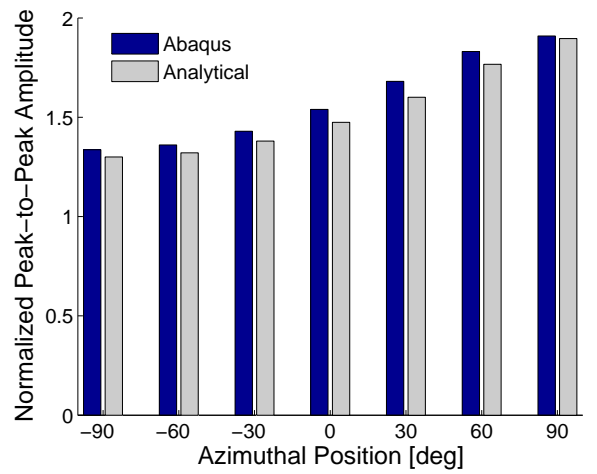

(b)

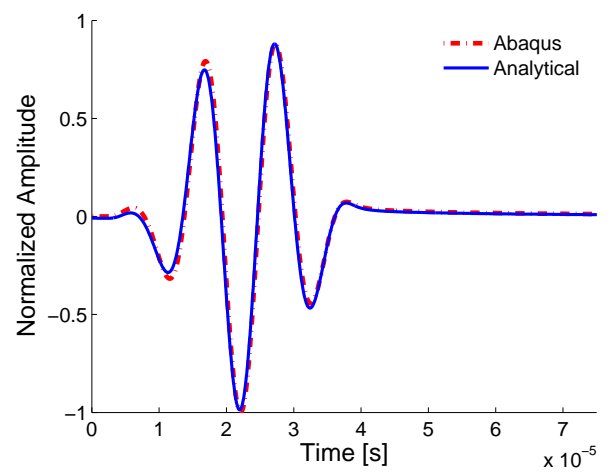

(d)

Figure 8. Comparison between FE and analytical results in regions I and II. (a) Points selected for comparison; (b) Amplitude comparison for antisymmetric $u_{3}$ in region I; (c) Amplitude comparison for antisymmetric $u_{3}$ in region II; (d) Sample time history for out-of-plane displacement at the origin.

would not be representative since this has been chosen as the metric. The results for both modes show that there are specific excitation frequencies at which the displacement amplitude is locally maximized. Similarly, there are frequencies that should be avoided due to the very small displacements induced by the transducer.

Another important observation is that, depending on the frequency at which the transducer is excited, a particular radial dimension is optimal for each mode and substrate thickness. The $\mathrm{S}_{0}$ mode shows the most variation in this regard, but it is clear that over most frequencies the cases of $\Delta r_{a} / b$ of 5 and 10 are superior to the rest. Similarly, it is seen that in the $\mathrm{A}_{0}$ mode, after a frequency of about $300 \mathrm{kHz}$, the case of $\Delta r_{a} / b$ of 2 has a larger amplitude than the rest. This result is important because the frequency of excitation is a critical parameter in the overall performance of the damage identification system. In actual SHM applications, different wavelengths (and therefore different frequencies) may be necessary to identify different damage types. For instance, the work of Cawley and Alleyne ${ }^{18}$ showed that for the case of throughthe-thickness notches (used to simulate surface cracks), Lamb waves were successful in their identification if the wavelength to notch depth ratio was in the order of 40. In addition, specific frequencies of excitation may be required to minimize dispersion. ${ }^{15}$ This phenomenon can result in excessive attenuation and spreading of the signal (both in space and time), thereby preventing adequate damage detection.

There are two effects associated with increasing the wedge angle. First, it can be seen that the difference between any two adjacent peaks increases for all radial dimensions and both modes. Secondly, as this dimension is increased, the behavior of the nodes (frequencies at which small displacements are obtained) intensifies, with the limiting case of a ring having a large number of such points with negligible displacement amplitude. This result shows an advantage of using a wedge-shaped APT instead of the more conventional 
ring configuration.

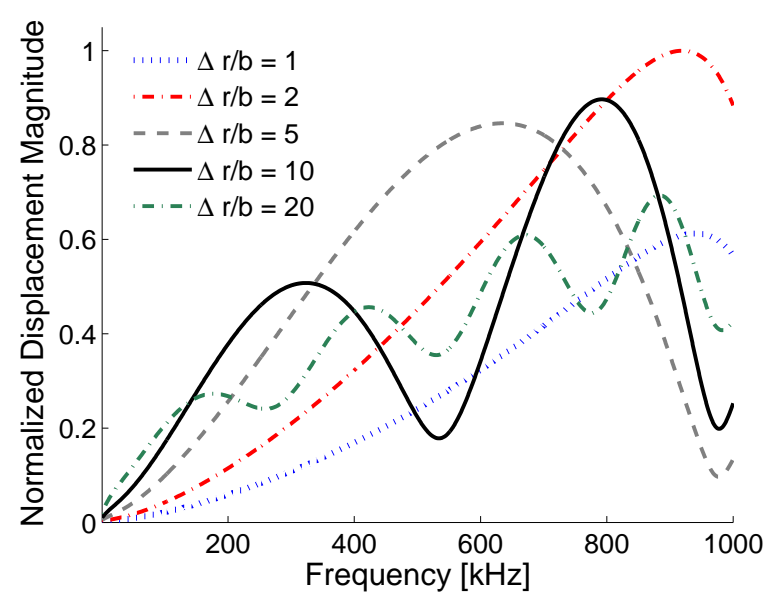

(a)

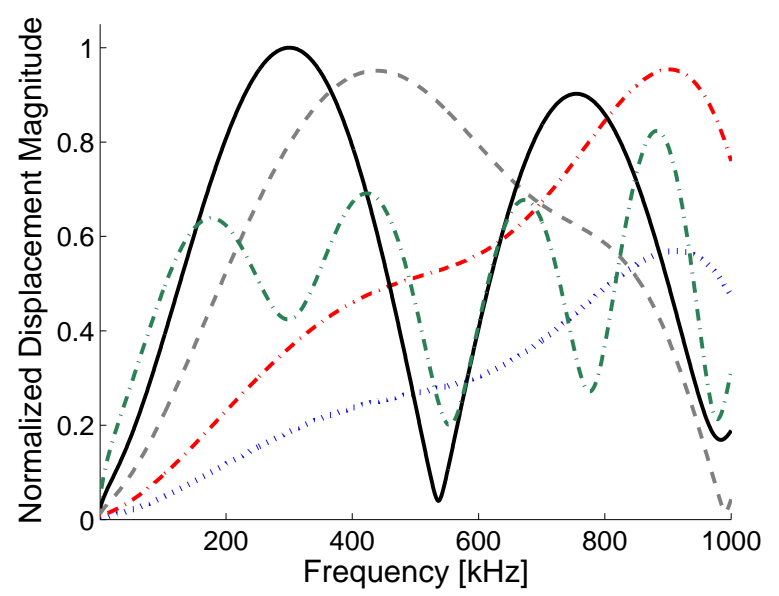

(c)

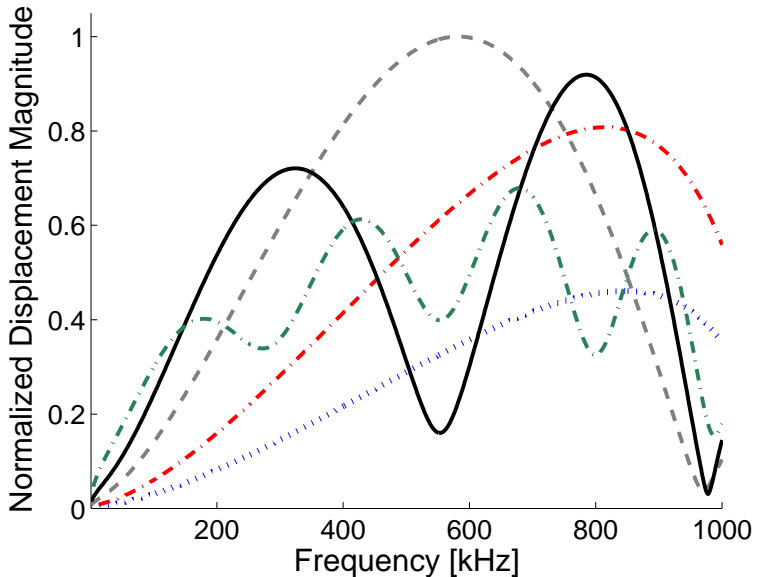

(b)

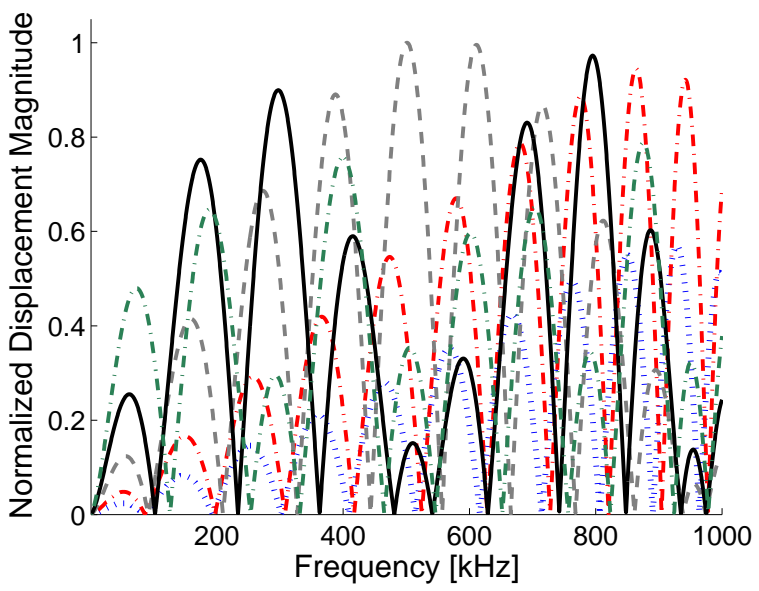

(d)

Figure 9. Effect of transducer dimensions on induced out-of-plane displacement field strength $\left(\mathrm{S}_{0}\right)$. $\Delta \theta=30^{\circ}$; (b) $\Delta \theta=60^{\circ}$; (c) $\Delta \theta=90^{\circ}$; (d) $\Delta \theta=360^{\circ}$.

\section{B. Focusing and Directionality}

As explained in section III, the displacement field is proportional to the force per unit length, $\tau_{0}$, exerted along the radial edges of the APT. This leads to the intuitive conclusion that actuators with longer edges receive a larger "net force". In the case of a wedge-shape geometry, the limiting case is a ring configuration where the length of the radial edge is maximized for fixed inner and outer radii. In this section, we seek to relate the force per unit length to an electric input metric to explore the increase in performance that can be obtained by the wedge-shaped APT. To do this, the piezoelectric strain induced by the APT must be considered.

Several approaches have been proposed to model the interaction between a structural substrate with a surface-bonded piezoelectric actuator under static conditions. This type of analysis generally employs an assumed strain field for the substrate, while the actuator is typically modeled as having a constant through-the-thickness strain. Crawley and de Luis ${ }^{9}$ considered this type of problem using a linear strain distribution for the substrate, which was modeled as an Euler-Bernoulli beam. Their analysis, however, consisted of two surface bonded piezoelectric actuators which resulted in a symmetric system. Chaudry and 


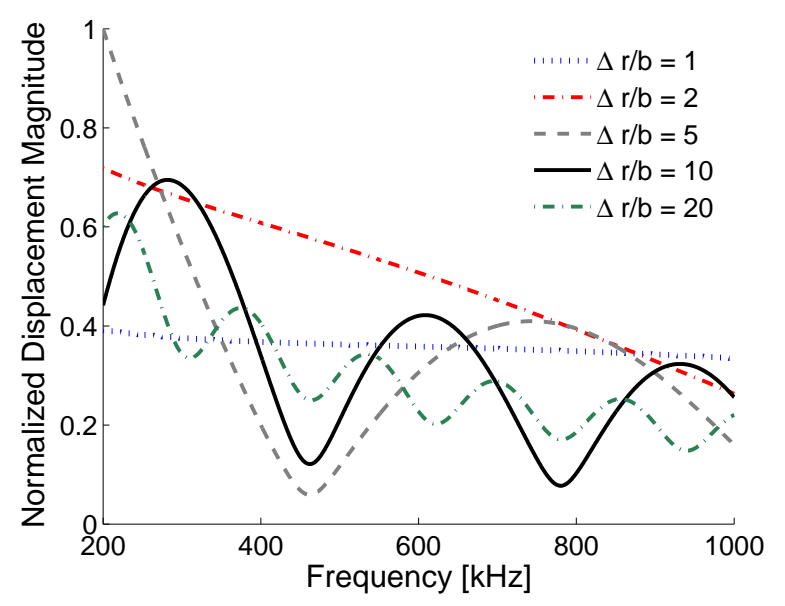

(a)

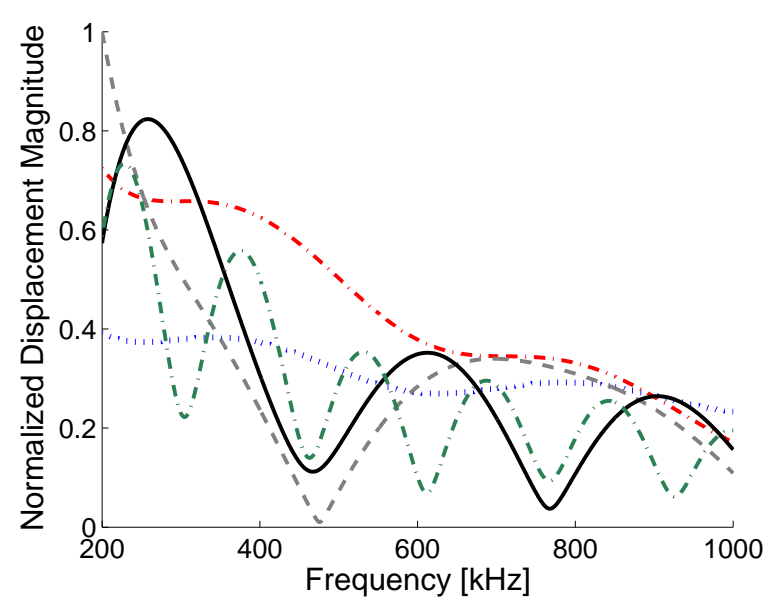

(c)

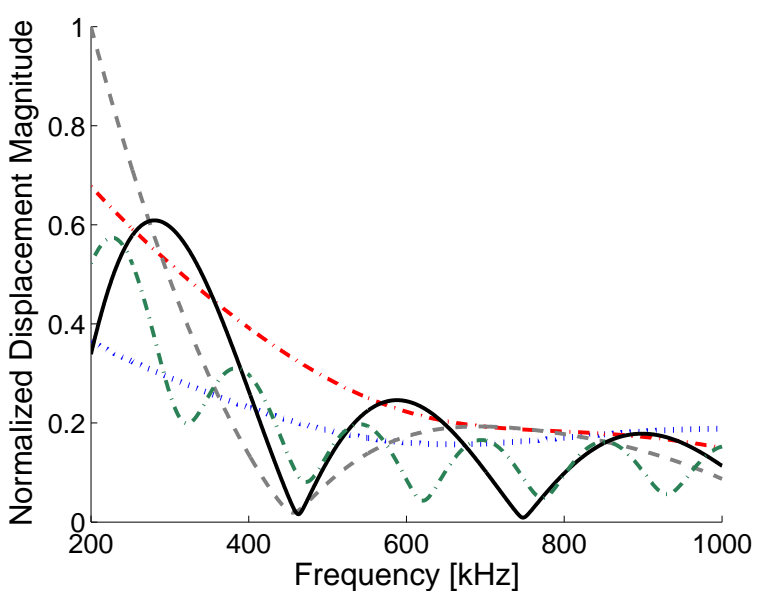

(b)

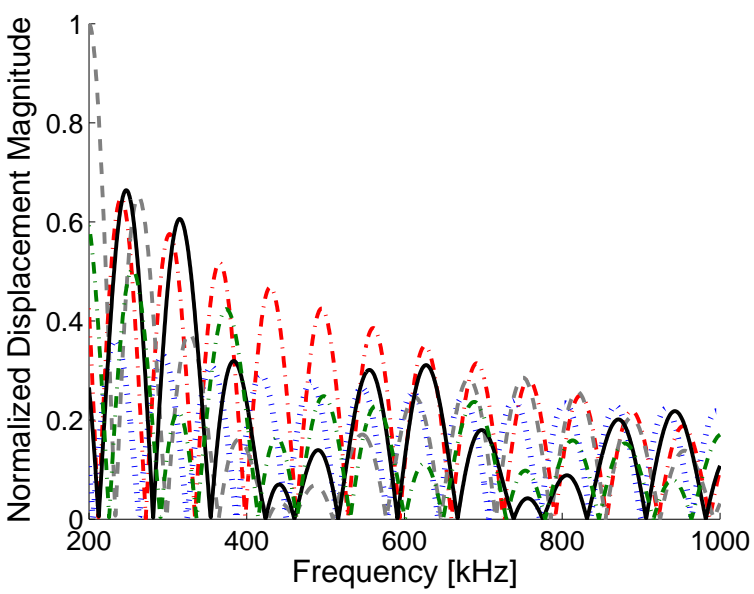

(d)

Figure 10. Effect of transducer dimensions on induced out-of-plane displacement field strength $\left(A_{0}\right)$. (a) $\Delta \theta=30^{\circ}$; (b) $\Delta \theta=60^{\circ}$; (c) $\Delta \theta=90^{\circ}$; (d) $\Delta \theta=360^{\circ}$.

Rogers ${ }^{19}$ conducted a similar study using a linear strain distribution for the actuator and substrate under the assumption of perfect bonding. They provided an expression for the curvature induced on the substrate by a single surface-bonded actuator, from which the force per unit length could be determined using the substrate's cross-sectional properties. In order to obtain a functional relationship between the force per unit length and the input voltage, this result will be employed here. It must be emphasized that this is an approximation as the mechanics of the substrate in this work are not those of Euler-Bernoulli beams. Then, for a rectangular cross-section, the force per unit length induced along the edges of the actuator can be expressed as:

$$
\tau_{0}=\frac{4 E_{B} b T(1+T) \psi}{6 T+4 T^{2}+4+\psi T^{2}+1} \epsilon_{P}
$$

where $T$ is defined by:

$$
T=\frac{2 b}{\bar{t}_{a}}
$$

and $\psi$ represents the stiffness-thickness ratio given by: 


$$
\psi=\frac{2 E_{B} b}{E_{a} \bar{t}_{a}}
$$

Finally, the piezoelectric strain can be expressed through:

$$
\epsilon_{P}=d_{33} \frac{V}{d_{I D E}}
$$

where $d_{I D E}$ represents the center-to-center distance between any two fingers in the APT interdigitated electrode. Note that Eq. (52) implies that a uniform electric field through the radial length of the actuator is assumed. In actual APT devices this is not the case since the electric field lines originate and end at the interdigitate electrode fingers. Since the field lines must be normal to the surfaces where they originate and end, there exists a small region close to the electrode fingers where the lines are not oriented along the fiber direction. Nevertheless, it has been shown that this is a reasonable simplification if the distance between any two electrode fingers, $d_{I D E}$, is sufficiently large. For instance, the work of Nguyen and Kornmann ${ }^{20}$ concluded that a minimum electrode spacing of $1.1 \mathrm{~mm}$ would be required to obtain a homogenous electric field along the length of the fiber for AFCs. If the APT is modeled as a capacitor with capacitance $C$, then a suitable metric for the electric input is the electric current drawn. For a harmonic input voltage of amplitude $V$ at a frequency $\omega$, the magnitude of the electric current is given by:

$$
I=\omega C V
$$

At this point, it is necessary to determine the dependence of the APT capacitance on the transducer's radial and azimuthal dimensions. The capacitance of this type of transducer is typically analyzed using the repetitive nature of the interdigitated electrode pattern. In this way, only the electric field in a representative unit cell, defined as the region between any two electrode fingers, is considered. A comprehensive study on this electric field was conducted by Lloyd ${ }^{21}$ using conformal mapping techniques. In that work, it was shown that the capacitance of each unit cell was primarily determined by the piezoceramic thickness, electrode finger width, and electrode finger spacing, while it scaled linearly with electrode finger length. Thus, the capacitance of a unit cell can be expressed as:

$$
C_{u c}=p\left(k, \epsilon_{0}, d_{I D E}, \bar{t}_{a}\right) L_{I D E}
$$

where, in the case of a wedge-shaped APT, the electrode finger length is determined by its radial position, $r_{I D E}$, and the transducer's azimuthal span, $\Delta \theta_{a}$, so that:

$$
C_{u c}=p\left(k, \epsilon_{0}, d_{I D E}, \bar{t}_{a}\right) r_{I D E} \Delta \theta_{a}
$$

A closed form expression for the function $p$ cannot be readily obtained. However, the work of Lloyd ${ }^{21}$ showed that this function is non-linearly dependent on the electrode finger and unit cell geometry, and that it increases exponentially as the center-to-center distance between the electrode fingers approaches zero. It is also important to note that any contribution from the capacitance of the epoxy has been neglected. This is a logical choice since its dielectric constant ${ }^{22}(k \sim 6)$ is much smaller than that for a typical piezoelectric ceramic $^{12}$ ( $k \sim 1700$, PZT-5A). The capacitance of the overall device may be obtained by considering it as a composition of capacitors connected in parallel. Then, the capacitance of each unit cell may be simply added together which results in:

$$
C=p\left(k, \epsilon_{0}, d_{I D E}, \bar{t}_{a}\right) \Delta \theta_{a} \frac{R_{O}+R_{I}}{2} \Delta r
$$

The results given by Eqs. (49), (52) and (56) combined with the definition of Eq. (53), indicate that the current drawn by the actuator will be linearly proportional to the product of the force per unit length, $\tau_{0}$, and the APT capacitance. This result further indicates that the actuator with largest angular span will draw the largest current in order to maintain a constant $\tau_{0}$.

Even though the ring-configuration draws the largest current according to the conclusion above, there are several shortcomings with its performance. First, the GW field excited has the same amplitude in every direction and therefore no focusing is possible. Secondly, as shown by the parametric results in the previous section, the ring geometry has a large number of frequency nodes that yield negligible displacement amplitudes. The wedge-shape APT offers a solution to both of these problems, especially if the same current 
input as in the case of the ring is utilized. Due to its geometry, this configuration is able to concentrate a significant portion of the excitation along the intended scan direction. Similarly, due to its shorter azimuthal span, the larger current input yields a larger magnitude for $\tau_{0}$, and hence an increased displacement amplitude everywhere. This effect can be quantified using the relationships provided by Eqs. (53) and (56). Equating the currents drawn by the ring-shaped and wedge-shaped APT devices yields:

$$
\omega p \Delta \theta \frac{R_{O}+R_{I}}{2} \Delta r V^{w e d g e}=\omega p 2 \pi \frac{R_{O}+R_{I}}{2} \Delta r V^{r i n g}
$$

from which, according to Eqs. (49) and (52), it follows that:

$$
\frac{\tau_{0}^{\text {wedge }}}{\tau_{0}^{\text {ring }}}=\frac{2 \pi}{\Delta \theta_{a}}
$$

The result from this analysis is presented in Fig. 11. This figure illustrates the magnitude of the harmonic out-of-plane displacement field obtained at $100 \mathrm{kHz}$ for several APT azimuthal spans. These results have been normalized at each point by the corresponding ring displacement. The transducer and substrate properties are similar to those in Table 1. The enhanced performance of the wedge-shaped transducer is clear as it produces displacements that are, for this particular frequency, twice as large as those induced by the ring. The white lines included in every plot indicate the azimuthal position where the displacements are equal to those induced by the ring. It is clear that as the wedge angle decreases, the energy concentrated along the intended scan direction increases. It must be emphasized that the purpose of this quantitative result is to illustrate the enhanced performance of the wedge-shaped configuration. It is clear from the parametric studies that, if excited at a frequency corresponding to a ring node, the wedge-shaped APTs will induce much larger displacements than the ring.

\section{Sensor Response}

In GW-based testing, a stress wave is excited by the piezoelectric actuator through the structural element whose condition is to be inspected. This wave typically experiences changes in its amplitude, frequency content, and group speed due to its interaction with any defects it encounters (e.g., cracks, corrosion, delaminations). Furthermore, these interactions produce additional waves that, generally, scatter in every direction. This process leads to the use of two primary methods of testing: the pitch-catch and pulseecho methods. The pitch-catch method is based on identifying damage based on the changes that defects introduce into the wave by locating a sensor a certain distance away from the actuator and recording the received wave. In contrast, the pulse-echo method uses the reflections scattered from defects to identify and locate damage. This typically involves using a transducer that acts both as actuator and sensor. In either method, information about the damage is obtained from strains sensed by the piezoelectric transducer and the corresponding voltage signal generated through the inverse piezoelectric effect. This voltage signal is then used to determine information about damage presence, location, and severity using adequate signal processing techniques. Therefore, it is necessary to relate the strains sensed by the transducer to the induced voltage. This will allow us to identify excitation frequencies and transducer dimensions that maximize the sensor response. Raghavan and Cesnik ${ }^{3}$ proposed a model to do this by modeling the sensor as a capacitor. Using the assumption that the sensor is under plane stress conditions, the following expression was presented:

$$
V_{s}=\frac{k \epsilon_{0} E_{s} \bar{t}_{s} g}{\left(1-\nu_{s}\right) C_{s}} \int_{A_{s}} \epsilon_{i i} d A
$$

where the piezoelectric constant to be used depends on the relative directions of the applied electric field and the induced strain. Similarly, the capacitance of the sensor, $C_{s}$, will depend on whether it is a uniform piezo material or an APT. An implication of this model is that the sensor is assumed to be infinitely compliant, so that it does not disturb the GW field produced by the actuator. The strains necessary to obtain the sensor response can be obtained from the displacement components defined previously by means of a linear strain-displacement relation. In polar coordinates, this is expressed as:

$$
\epsilon_{r r}=\frac{\partial u_{r}}{\partial r}
$$



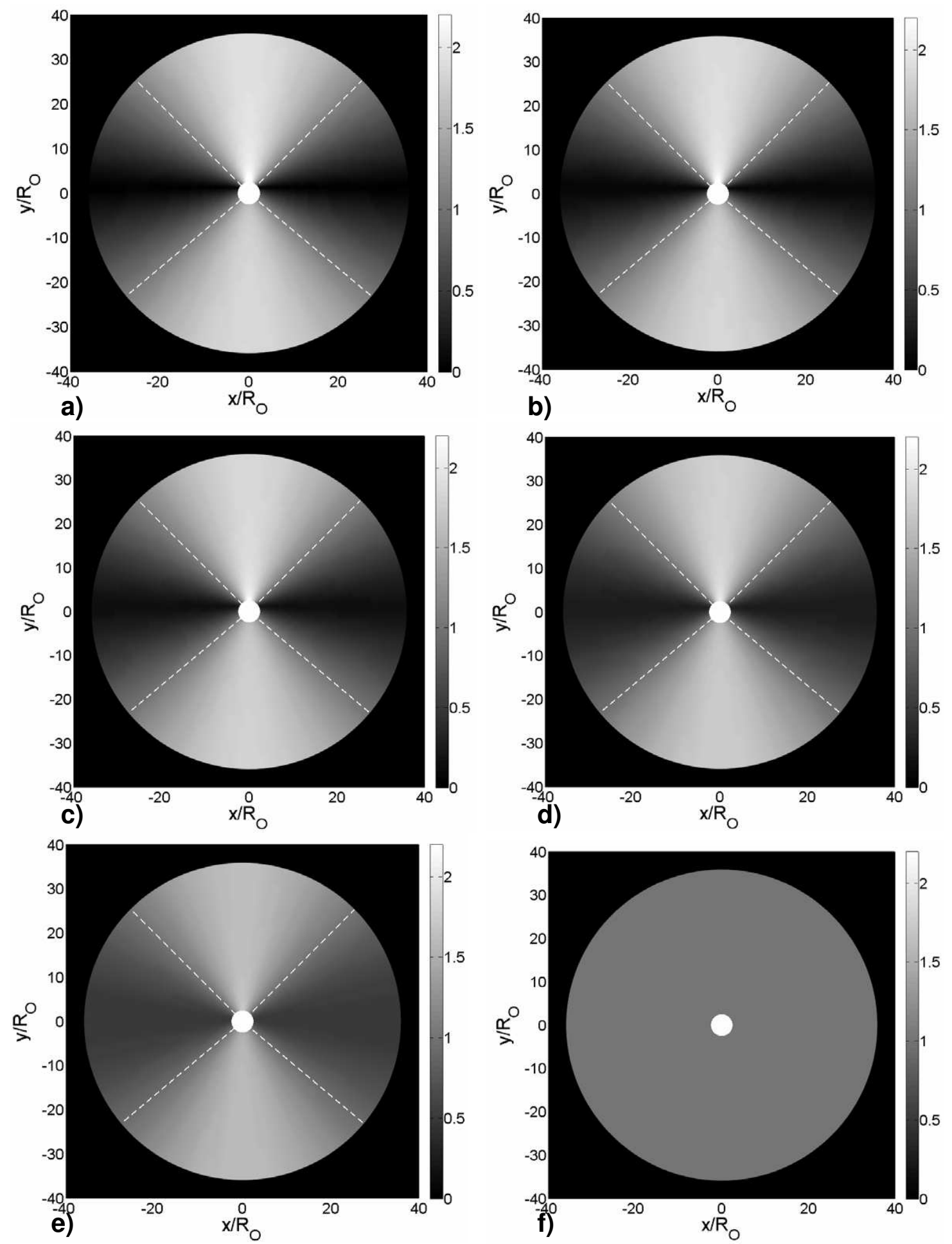

Figure 11. Harmonic out-of-plane displacement field $\left(S_{0}\right)$ for a frequency of $100 \mathrm{kHz}$ using ring power and the following actuator wedge angles: (a) $15^{\circ}$; (b) $30^{\circ}$; (c) $45^{\circ} ;$ (d) $60^{\circ}$; (e) $90^{\circ}$; (f) $360^{\circ}$. The dashed lines show the locations where the GW amplitude is the same for both the wedge-shaped and ring transducers. 


$$
\epsilon_{\theta \theta}=\frac{u_{r}}{r}+\frac{1}{r} \frac{\partial u_{\theta}}{d \theta}
$$

where $u_{r}$ and $u_{\theta}$ are the radial and azimuthal displacement components, respectively. These components can be expressed in terms of the cartesian components obtained previously through the transformation:

$$
\left\{\begin{array}{l}
u_{r} \\
u_{\theta}
\end{array}\right\}=\left[\begin{array}{cc}
\cos \theta & \sin \theta \\
-\sin \theta & \cos \theta
\end{array}\right]\left\{\begin{array}{l}
u_{1} \\
u_{2}
\end{array}\right\}
$$

Since the cartesian components were previously obtained in terms of polar coordinates, it follows from Eq. (62) that:

$$
\frac{\partial u_{r}}{\partial r}=\frac{\partial u_{1}}{\partial r} \cos \theta+\frac{\partial u_{2}}{\partial r} \sin \theta
$$

and

$$
\frac{\partial u_{\theta}}{\partial \theta}=\cos \theta\left(\frac{\partial u_{2}}{\partial \theta}-u_{1}\right)-\sin \theta\left(\frac{\partial u_{1}}{\partial \theta}+u 2\right)
$$

Similarly, the strain-displacement relation in cartesian coordinates is given by:

$$
\begin{aligned}
& \epsilon_{x x}=\frac{\partial u_{1}}{\partial x} \\
& \epsilon_{y y}=\frac{\partial u_{2}}{\partial y}
\end{aligned}
$$

Since the displacement components were found in terms of polar coordinates, the coordinate transformation $r=\sqrt{x^{2}+y^{2}}, \theta=\tan ^{-1}(y / x)$ is used along with the chain rule of derivatives to obtain:

$$
\begin{aligned}
\epsilon_{x x} & =\frac{\partial u_{1}}{\partial r} \cos \theta-\frac{\partial u_{1}}{\partial \theta} \frac{\sin \theta}{r} \\
\epsilon_{y y} & =\frac{\partial u_{2}}{\partial r} \sin \theta+\frac{\partial u_{2}}{\partial \theta} \frac{\cos \theta}{r}
\end{aligned}
$$

Finally, the $r$ and $\theta$ derivatives of the displacement components, for the antisymmetric mode, may be expressed in vector form as:

$$
\frac{d \mathbf{u}_{A}}{d r}=\sum_{k=-\infty}^{\infty}\left[\frac{i \pi^{2} \xi_{A} \boldsymbol{\Gamma}_{A}\left(\xi_{A}\right)}{\zeta_{k}(\theta) D_{A}^{\prime}\left(\xi_{A}\right)} \boldsymbol{\Delta}_{A}\left(k, \xi_{A}\right)\left\{H_{k-1}^{(2)}\left(\xi_{A} r\right)-H_{k+1}^{(2)}\left(\xi_{A} r\right)\right\}\right]
$$

and:

$$
\frac{d \mathbf{u}_{A}}{d \theta}=\sum_{k=-\infty}^{\infty}\left[\frac{-2 k \pi^{2} \boldsymbol{\Gamma}_{A}\left(\xi_{A}\right)}{\zeta_{k}(\theta) D_{A}^{\prime}\left(\xi_{A}\right)} \boldsymbol{\Delta}_{A}\left(k, \xi_{A}\right) H_{k}^{(2)}\left(\xi_{A} r\right)\right]
$$

\section{A. Wedge-shaped APT sensor}

The response of a wedge-shaped APT sensor under the GW field excited by a wedge-shaped APT actuator is now derived. This analysis is based on the assumption that only extensional strains along the piezoelectric fiber direction are sensed. This is a reasonable simplification since the induced shear strains are small compared to the extensional ones. Furthermore, as discussed in section III, the piezoelectric performance of the transducer along the normal direction to the fiber is very weak. Therefore, for the wedge-shaped configuration, only $\epsilon_{r r}$ is needed. Consider a wedge-shaped APT sensor centered at the point $r=r_{s}, \theta=\theta_{s}$, with radial dimension $\Delta r_{s}$, angular span $\Delta \theta_{s}$, and surface area $A_{s}=r_{s} \Delta r_{s} \Delta \theta_{s}$, as shown in Fig. 12(a). The sensor is subjected to the GW field excited by a wedge-shaped APT actuator with radial dimension $\Delta r_{a}$ and angular span $\Delta \theta_{a}$ centered at the point $r=0.5\left(R_{I}+R_{O}\right), \theta=90^{\circ}$. Using the expression for the APT capacitance presented in section VI, the voltage induced in the APT due to the strains sensed is given by: 


$$
V_{s}=\frac{k \epsilon_{0} E_{s} \bar{t}_{s} g_{33}}{\left(1-\nu_{s}\right) p A_{s}} \int_{\theta_{s}-\frac{\Delta \theta_{s}}{2}}^{\theta_{s}+\frac{\Delta \theta_{s}}{\theta_{s}}} \int_{r_{s}-\frac{\Delta r_{s}}{2}}^{r_{s}+\frac{\Delta r_{s}}{2}} \epsilon_{r r} r d r d \theta
$$

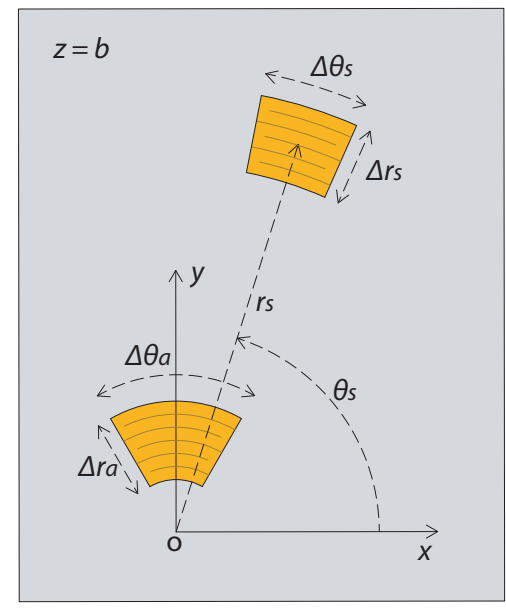

(a) Wedge-shaped APT sensor

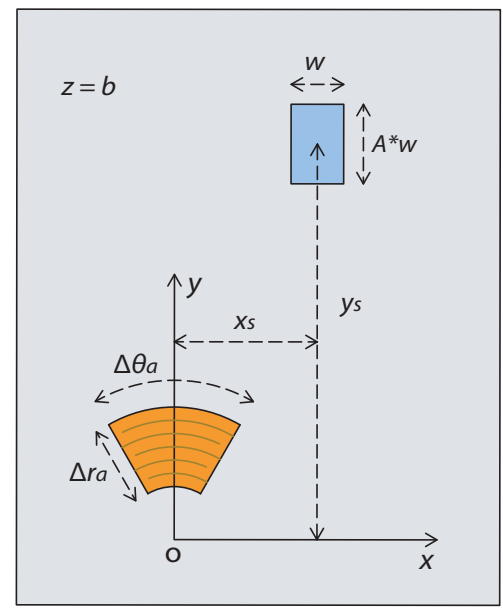

(b) Rectangular piezo sensor

Figure 12. Sensor geometry and location used in analysis.

where $p$ is the function introduced in Eq. (56). Note that in the case of an APT, $g_{33}$ is used. Using the definition in Eq. (71), the voltage is given by:

$$
V_{s}=\frac{k \epsilon_{0} E_{s} \bar{t}_{s} g_{33}}{\left(1-\nu_{s}\right) p A_{s}} \int_{\theta_{s}-\frac{\Delta \theta_{s}}{2}}^{\theta_{s}+\frac{\Delta \theta_{s}}{2}} \int_{r_{s}-\frac{\Delta r_{s}}{2}}^{r_{s}+\frac{\Delta r_{s}}{2}}\left[\frac{\partial u_{1}}{\partial r} \cos \theta+\frac{\partial u_{2}}{\partial r} \sin \theta\right] r d r d \theta
$$

Note that Eq. (72) holds for harmonic forcing at a frequency $\omega$. For the general case of a time-dependent excitation, such as the toneburst signal considered before, the integrand is multiplied by the time Fourier transform of this signal and integrated over an infinite frequency range, as outlined in section IV. However, in order to identify optimal excitation frequencies and transducer dimensions a harmonic analysis is sufficient. Once these have been identified, they can be used as center frequencies of time-dependent excitations.

The variation in three parameters was examined. First, we explored the excitation frequencies to determine values at which the sensor response would be maximum, as well as to identify values that should be avoided due to negligible sensing response. Secondly, the ratio of the sensor to actuator size was investigated to determine optimal dimensions. This analysis was performed in two separate steps; the ratio of the radial dimension was first explored by defining the non-dimensional parameter $\mathrm{r}^{*}$ as the ratio $\Delta r_{s} / \Delta r_{a}$ and obtaining the sensor response, given by Eq. (72), over a wide frequency range for both symmetric and antisymmetric modes. The result from this analysis is shown in Fig. 13. The location of the sensor was set to $r_{s}=15 R_{O}$ and $\theta_{s}=90^{\circ}$; this azimuthal position was selected since it coincides with the transducer's centerline which is the intended scanning direction. The actuator dimensions were similar to the ones presented in Table 1. In these and subsequent sensor response plots, the results presented are normalized by the product of the force per unit length, $\tau_{0}$, and the function $p$, both of which are constant values for a given APT geometry. In addition, the curves are scaled by the maximum value in each set.

It is clear that the ratio of the radial dimension greatly affects the response, with smaller sensor sizes producing better results. In addition to the decrease in amplitude observed for larger sensors, it can also be seen that the number of nodes increases significantly. The two modes show different qualitative behavior over the range of frequencies studied with the $\mathrm{A}_{0}$ mode showing two main frequencies at which high response is obtained, as opposed to the $S_{0}$ case which shows primarily one up to $500 \mathrm{kHz}$.

The effect of varying the ratio of azimuthal spans was also examined, which resulted in the definition of the parameter $\theta^{*}$ as $\Delta \theta_{s} / \Delta \theta_{a}$. The results from this analysis, for $\mathrm{r}^{*}$ fixed at 1 , are shown in Fig. 14 . As it 


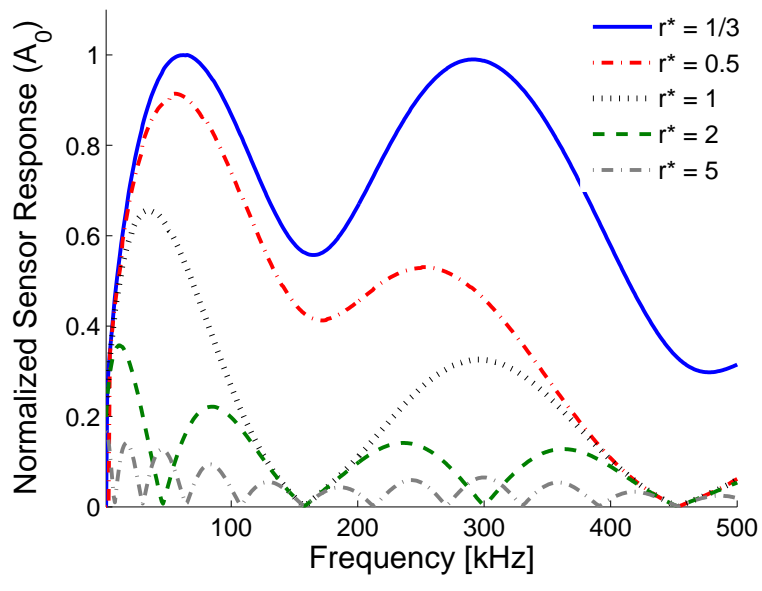

(a) $\mathbf{A}_{0}$ mode

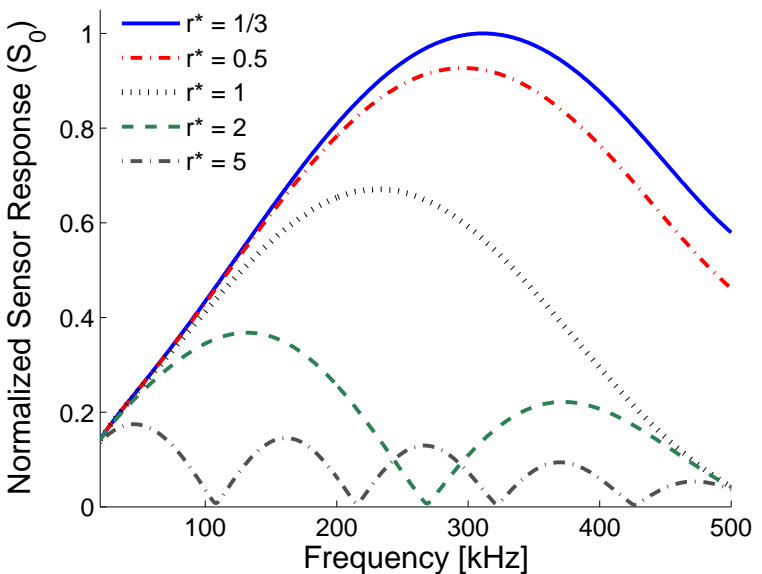

(b) $\mathbf{S}_{0}$ mode

Figure 13. Effect of radial dimension on wedge-shaped APT sensor response. The baseline case was $\Delta r_{a}=0.005$ m.

can be seen, this characteristic does not affect the trend observed in frequency (Fig. 13), but instead causes a decrease in amplitude. Notice that this effect is significant only if the actuator is made much smaller than the sensor or vice-versa. These trends are logical since it is expected that changing the sensor dimension in the wave propagation direction will have the most significant effect.

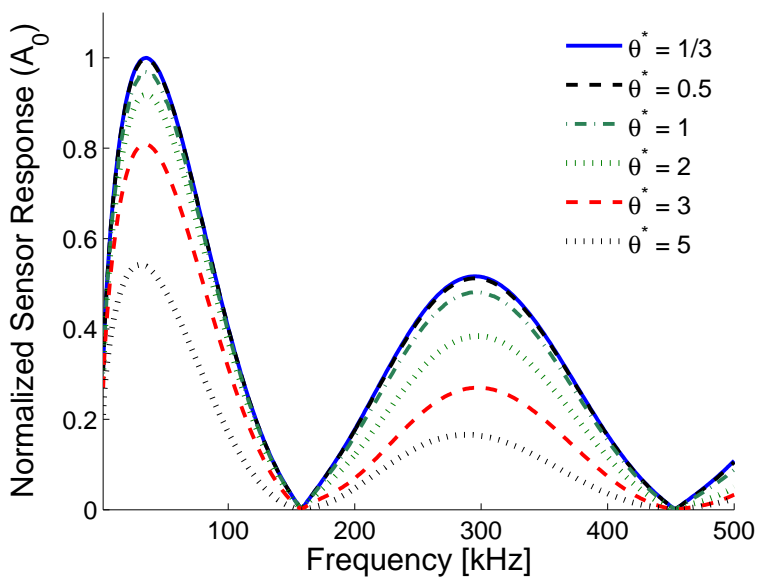

(a) $\mathbf{A}_{0}$ mode

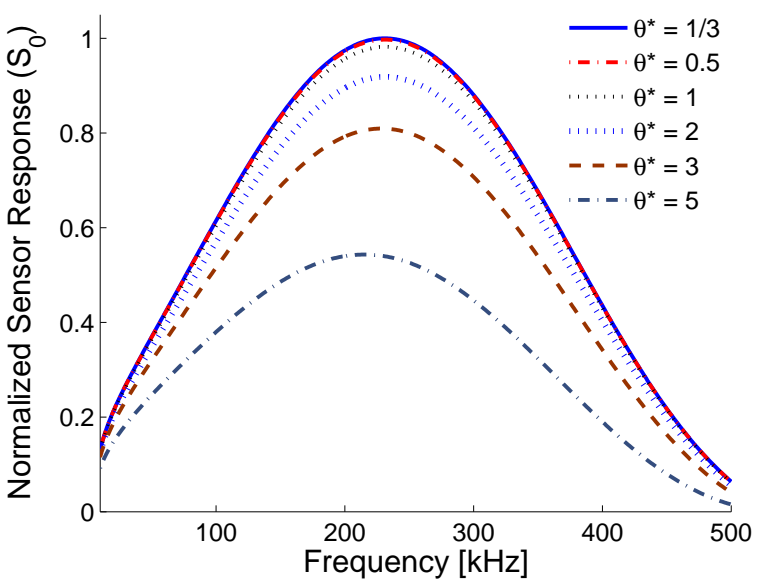

(b) $\mathbf{S}_{0}$ mode

Figure 14. Effect of azimuthal dimension on wedge-shaped APT sensor response. The baseline case was $\Delta r_{a}=0.005 \mathrm{~m}, \mathrm{r}^{*}=1$.

\section{B. Rectangular piezo sensor}

The sensor response of a homogeneous rectangular piezoelectric sensor is also of practical interest. Consider a rectangular sensor of width $w$, and aspect ratio $A^{*}$, so that, $h=A^{*} w$ and $A_{s}=A^{*} w^{2}$, as shown in Fig. 12(b). The sensor is centered at the point $x=x_{s}, y=y_{s}$ and is under the GW field excited by a wedgeshaped APT of radial span $\Delta r_{a}$ and angular span $\Delta \theta_{a}$ centered at the point $r=0.5\left(R_{I}+R_{O}\right), \theta=\theta_{a}$. 
In contrast to the case of an APT sensor, the piezo material has isotropic piezoelectric properties in the plane normal to the poling direction, and therefore senses all in-plane extensional strains. In addition, this enables the piezo to be modeled as a parallel plate capacitor filled with a dielectric material. In this way, its capacitance may be expressed through:

$$
C_{s}=\frac{k \epsilon_{0} A_{s}}{\bar{t}_{s}}
$$

Consequently, its sensor response is given by: ${ }^{3}$

$$
V_{s}=\frac{E_{s} \bar{t}_{s} g_{13}}{A_{s}\left(1-\nu_{s}\right)} \int_{y_{s}-A^{*} \frac{w}{2}}^{y_{s}+A^{*} \frac{w}{2}} \int_{x_{s}-\frac{w}{2}}^{x_{s}+\frac{w}{2}}\left(\epsilon_{x x}+\epsilon_{y y}\right) d x d y
$$

where $\epsilon_{x x}$ and $\epsilon_{y y}$ are defined by Eqs. (67) and (68), respectively. As in the case of a wedge-shaped APT, two sensor dimensions were explored. First, the width of the sensor was considered, which resulted in the definition of the non-dimensional parameter $\mathrm{w}^{*}$ as $w / \Delta r_{a}$. The effect of the sensor's aspect ratio was also studied. The results from this analysis are shown in Figs. 15 and 16. In the study of the aspect ratio, the value of $\mathrm{w}^{*}$ was kept fixed at 1 . Similar qualitative differences between the two modes are observed in this case, with the antisymmetric mode showing the highest response at a higher frequency than in the previous case. An important distinction between the two sensor types is that in the case of a rectangular piezo, both dimensions influence the observed trends in frequency. As in the previous case, it is evident that smaller sensors produce better results.

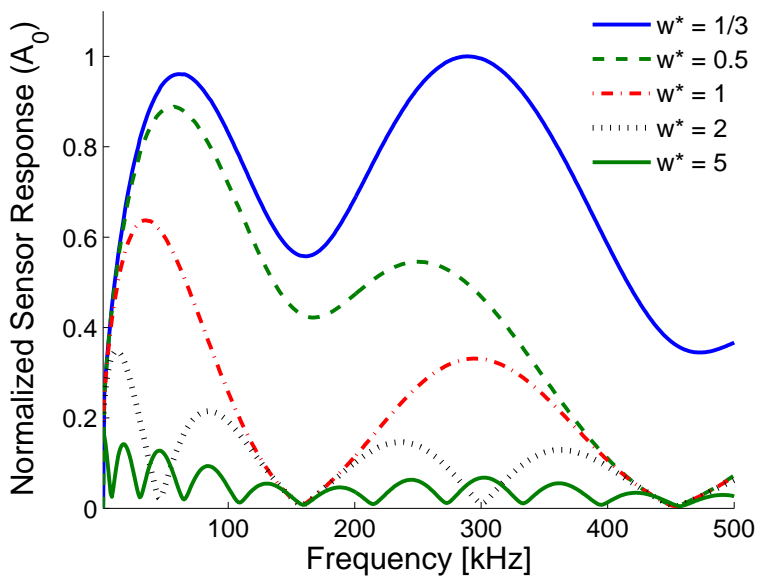

(a) $\mathbf{A}_{0}$ mode

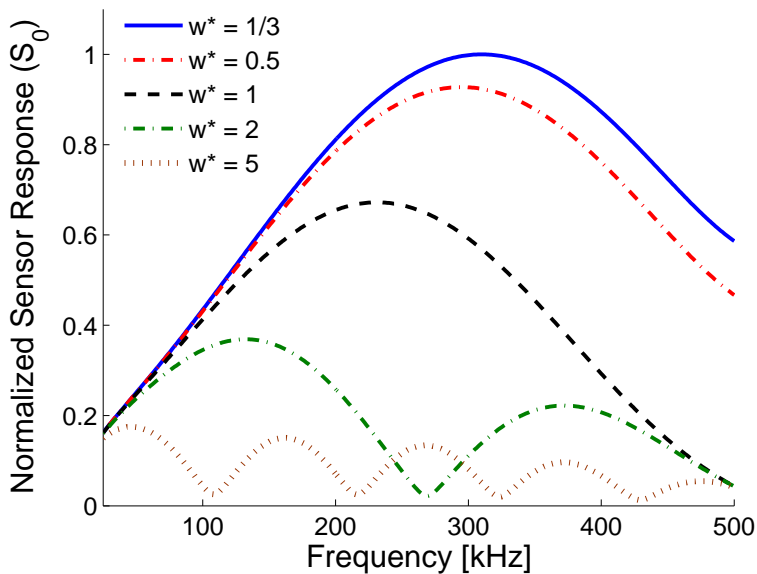

(b) $\mathrm{S}_{0}$ mode

Figure 15. Effect of sensor width on piezo sensor response. The baseline case was $\Delta r_{a}=0.005 \mathrm{~m}$.

\section{Concluding Remarks}

The guided wave (GW) field excited by a surface-bonded, wedge-shaped anisotropic piezocomposite transducer (APT) was investigated in this paper. The analysis considered uncoupled dynamics for the actuator and substrate, and their interaction was modeled as surface tractions along the actuator's edges. Analytical solutions were presented for three spatial regions, determined by the location of the transducer's radial edges. Results from three-dimensional finite element simulations demonstrated the spatial and temporal accuracy of the proposed solution. Parametric studies were conducted to explore how the actuator dimensions influenced the displacement field induced over a wide frequency range. Specific combinations of frequency and transducer dimensions that maximize the displacements obtained were identified. Likewise, combinations that should be avoided due to decreased response were also found. The results revealed that the ratio of the actuator radial dimension and substrate thickness greatly affects the actuator's performance. It was also 


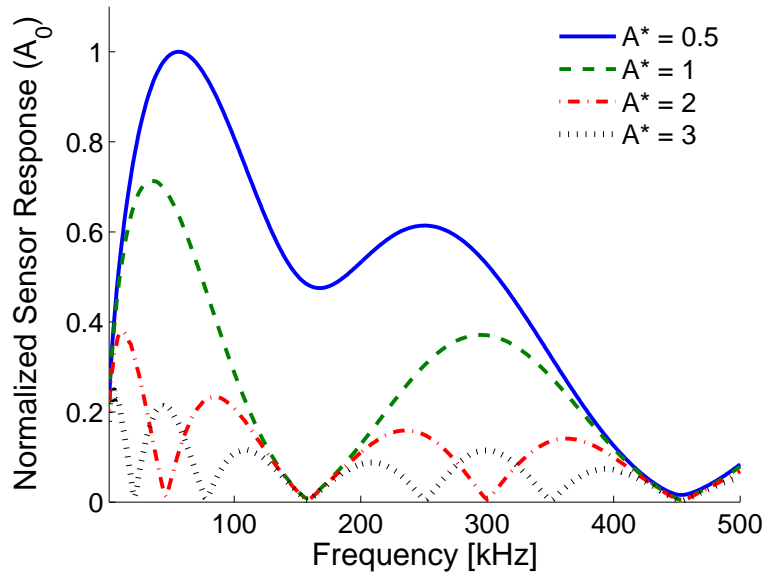

(a) $\mathbf{A}_{0}$ mode

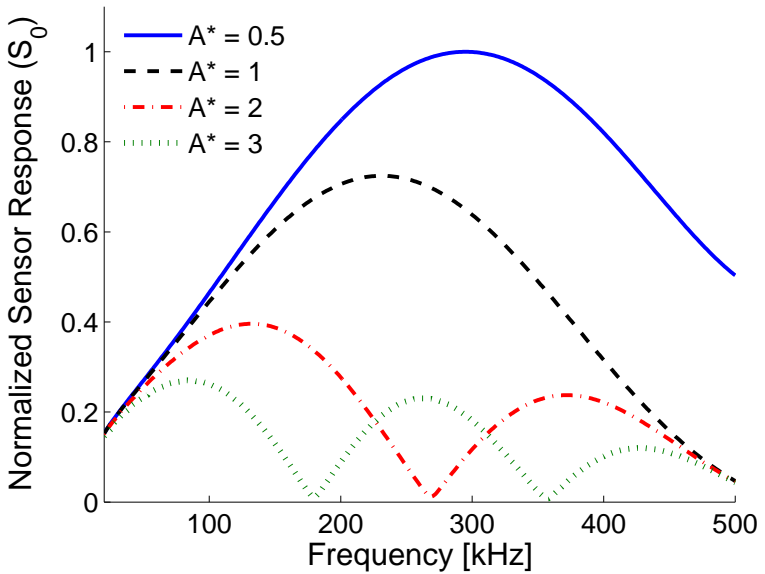

(b) $\mathbf{S}_{0}$ mode

Figure 16. Effect of sensor aspect ratio $\left(A^{*}\right)$ on piezo sensor response. The baseline case was $\Delta r_{a}=0.005 \mathrm{~m}$.

found that smaller actuator azimuthal spans yield better behavior in frequency. Employing a reduced structural theory for the substrate, and modeling the actuator as a capacitor, a functional relationship between the displacement amplitude and the current drawn by the transducer was obtained. This result was used to show (and quantify) that the wedge-shape is superior to the more conventional ring configuration, due to its ability to efficiently concentrate the actuation along the intended scan direction. The sensor response of a uniform rectangular piezoelectric wafer and a wedge-shaped APT under the GW field excited by a wedge-shaped APT was also investigated. This analysis was based on modeling the actuator as a capacitor. It was found that the radial dimension of the APT sensor significantly affects the response in frequency, while the azimuthal span only affects the response amplitude. The overall trend that smaller sensors produce enhanced response was confirmed. The results presented in this paper indicate that for a particular structural health monitoring application, where the frequency of excitation and substrate dimensions are known, specific actuator and sensor dimensions can be selected to maximize the system's performance. 


\section{Appendix}

The column vector of coefficients, $\boldsymbol{\Delta}$, first introduced in Eq. (35), is defined through:

$$
\boldsymbol{\Delta}=\left\{\begin{array}{c}
\varrho_{-2-k}^{(1)}-\varrho_{-k}^{(2)}-\varrho_{2-k}^{(3)} \\
\varsigma_{-2-k}^{(1)}-\varsigma_{-k}^{(2)}-\varsigma_{2-k}^{(3)} \\
\bar{\delta}_{1-k}^{(1)}+\bar{\delta}_{-1-k}^{(2)}
\end{array}\right\}
$$

where the individual components have the following definitions:

$$
\begin{gathered}
\varrho_{k}^{(1)}=\left[c_{k}^{(2)} \gamma_{5}^{(1)}-c_{k}^{(1)} \gamma_{4}^{(1)}\right](-i)^{k} \chi_{k} \\
\varrho_{k}^{(2)}=c_{k}^{(1)} \gamma_{3}^{(1)}(-i)^{k} \chi_{k} \\
\varrho_{k}^{(3)}=\left[c_{k}^{(1)} \gamma_{4}^{(1)}+c_{k}^{(2)} \gamma_{5}^{(1)}\right](-i)^{k} \chi_{k} \\
\varsigma_{k}^{(1)}=\left[c_{k}^{(1)} \gamma_{1}^{(2)}-c_{k}^{(2)} \gamma_{5}^{(2)}\right](-i)^{k} \chi_{k} \\
\varsigma_{k}^{(2)}=c_{k}^{(2)} \gamma_{4}^{(2)}(-i)^{k} \chi_{k} \\
\varsigma_{k}^{(3)}=\left[c_{k}^{(1)} \gamma_{1}^{(2)}+c_{k}^{(2)} \gamma_{5}^{(2)}\right](-i)^{k} \chi_{k} \\
\bar{\delta}_{k}^{(1)}=\frac{1}{2}\left(c_{k}^{(1)}+i c_{k}^{(2)}\right)(-i)^{k} \chi_{k} \\
\bar{\delta}_{k}^{(2)}=\frac{1}{2}\left(c_{k}^{(1)}-i c_{k}^{(2)}\right)(-i)^{k} \chi_{k}
\end{gathered}
$$

Similarly, the column vector $\boldsymbol{\Lambda}$ is defined as:

$$
\boldsymbol{\Lambda}=\left\{\begin{array}{c}
\eta_{-2-k}^{(1)}-\eta_{-k}^{(2)}-\eta_{2-k}^{(3)} \\
\kappa_{-2-k}^{(1)}-\kappa_{-k}^{(2)}-\kappa_{2-k}^{(3)} \\
\bar{\nu}_{1-k}^{(1)}+\bar{\nu}_{-1-k}^{(2)}
\end{array}\right\}
$$

where the individual components have the following definitions:

$$
\begin{aligned}
\eta_{k}^{(1)}= & {\left[c_{k}^{(2)} \gamma_{5}^{(1)}-c_{k}^{(1)} \gamma_{4}^{(1)}\right]\left[r_{O} H_{k}^{(2)}\left(\xi_{A} r\right)-r_{I} H_{k}^{(2)}\left(\xi_{A} r\right)\right](-i)^{k} } \\
& \eta_{k}^{(2)}=c_{k}^{(1)} \gamma_{3}^{(1)}\left[r_{O} H_{k}^{(2)}\left(\xi_{A} r\right)-r_{I} H_{k}^{(2)}\left(\xi_{A} r\right)\right](-i)^{k} \\
\eta_{k}^{(3)}= & {\left[c_{k}^{(1)} \gamma_{4}^{(1)}+c_{k}^{(2)} \gamma_{5}^{(1)}\right]\left[r_{O} H_{k}^{(2)}\left(\xi_{A} r\right)-r_{I} H_{k}^{(2)}\left(\xi_{A} r\right)\right](-i)^{k} } \\
\kappa_{k}^{(1)}= & {\left[c_{k}^{(1)} \gamma_{1}^{(2)}-c_{k}^{(2)} \gamma_{5}^{(2)}\right]\left[r_{O} H_{k}^{(2)}\left(\xi_{A} r\right)-r_{I} H_{k}^{(2)}\left(\xi_{A} r\right)\right](-i)^{k} } \\
& \kappa_{k}^{(2)}=c_{k}^{(2)} \gamma_{4}^{(2)}\left[r_{O} H_{k}^{(2)}\left(\xi_{A} r\right)-r_{I} H_{k}^{(2)}\left(\xi_{A} r\right)\right](-i)^{k} \\
\kappa_{k}^{(3)}= & {\left[c_{k}^{(1)} \gamma_{1}^{(2)}+c_{k}^{(2)} \gamma_{5}^{(2)}\right]\left[r_{O} H_{k}^{(2)}\left(\xi_{A} r\right)-r_{I} H_{k}^{(2)}\left(\xi_{A} r\right)\right](-i)^{k} } \\
\bar{\nu}_{k}^{(1)}= & \frac{1}{2}\left(c_{k}^{(1)}+i c_{k}^{(2)}\right)\left[r_{O} H_{k}^{(2)}\left(\xi_{A} r\right)-r_{I} H_{k}^{(2)}\left(\xi_{A} r\right)\right](-i)^{k} \\
\bar{\nu}_{k}^{(2)}= & \frac{1}{2}\left(c_{k}^{(1)}-i c_{k}^{(2)}\right)\left[r_{O} H_{k}^{(2)}\left(\xi_{A} r\right)-r_{I} H_{k}^{(2)}\left(\xi_{A} r\right)\right](-i)^{k}
\end{aligned}
$$

26 of 29 
The column vector $\widetilde{\Upsilon}$ is defined as:

$$
\tilde{\boldsymbol{\Upsilon}}=\left\{\begin{array}{c}
\tilde{\rho}_{-2-k}^{(1)}-\tilde{\rho}_{-k}^{(2)}-\tilde{\rho}_{2-k}^{(3)} \\
\tilde{\psi}_{-2-k}^{(1)}-\tilde{\psi}_{-k}^{(2)}-\tilde{\psi}_{2-k}^{(3)} \\
\tilde{v}_{1-k}^{(1)}+\tilde{v}_{-1-k}^{(2)}
\end{array}\right\}
$$

where the individual components are given by:

$$
\begin{gathered}
\tilde{\rho}_{k}^{(1)}=\left[c_{k}^{(2)} \gamma_{5}^{(1)}-c_{k}^{(1)} \gamma_{4}^{(1)}\right](-i)^{k} r_{O} H_{k}^{(2)}\left(\xi_{A} r_{O}\right) \\
\tilde{\rho}_{k}^{(2)}=c_{k}^{(1)} \gamma_{3}^{(1)}(-i)^{k} r_{O} H_{k}^{(2)}\left(\xi_{A} r_{O}\right) \\
\tilde{\rho}_{k}^{(2)}=\left[c_{k}^{(1)} \gamma_{4}^{(1)}+c_{k}^{(2)} \gamma_{5}^{(1)}\right](-i)^{k} r_{O} H_{k}^{(2)}\left(\xi_{A} r_{O}\right) \\
\tilde{\psi}_{k}^{(1)}=\left[c_{k}^{(1)} \gamma_{1}^{(2)}-c_{k}^{(2)} \gamma_{5}^{(2)}\right](-i)^{k} r_{O} H_{k}^{(2)}\left(\xi_{A} r_{O}\right) \\
\tilde{\psi}_{k}^{(2)}=c_{k}^{(2)} \gamma_{4}^{(2)}(-i)^{k} r_{O} H_{k}^{(2)}\left(\xi_{A} r_{O}\right) \\
\tilde{\psi}_{k}^{(3)}=\left[c_{k}^{(1)} \gamma_{1}^{(2)}+c_{k}^{(2)} \gamma_{5}^{(2)}\right](-i)^{k} r_{O} H_{k}^{(2)}\left(\xi_{A} r_{O}\right) \\
\tilde{v}_{k}^{(1)}=\frac{1}{2}\left(c_{k}^{(1)}+i c_{k}^{(2)}\right)(-i)^{k} r_{O} H_{k}^{(2)}\left(\xi_{A} r_{O}\right) \\
\tilde{v}_{k}^{(2)}=\frac{1}{2}\left(c_{k}^{(1)}-i c_{k}^{(2)}\right)(-i)^{k} r_{O} H_{k}^{(2)}\left(\xi_{A} r_{O}\right)
\end{gathered}
$$

Finally, the column vector $\Upsilon$ is defined as:

$$
\mathbf{\Upsilon}=\left\{\begin{array}{c}
\bar{\rho}_{-2-k}^{(1)}-\bar{\rho}_{-k}^{(2)}-\bar{\rho}_{2-k}^{(3)} \\
\hat{\psi}_{-2-k}^{(1)}-\hat{\psi}_{-k}^{(2)}-\hat{\psi}_{2-k}^{(3)} \\
v_{1-k}^{(1)}+v_{-1-k}^{(2)}
\end{array}\right\}
$$

where the individual components are given by:

$$
\begin{gathered}
\bar{\rho}_{k}^{(1)}=\left[c_{k}^{(2)} \gamma_{5}^{(1)}-c_{k}^{(1)} \gamma_{4}^{(1)}\right](-i)^{k} r_{I} J_{k}\left(\xi_{A} r_{I}\right) \\
\bar{\rho}_{k}^{(2)}=c_{k}^{(1)} \gamma_{3}^{(1)}(-i)^{k} r_{I} J_{k}\left(\xi_{A} r_{I}\right) \\
\bar{\rho}_{k}^{(2)}=\left[c_{k}^{(1)} \gamma_{4}^{(1)}+c_{k}^{(2)} \gamma_{5}^{(1)}\right](-i)^{k} r_{I} J_{k}\left(\xi_{A} r_{I}\right) \\
\hat{\psi}_{k}^{(1)}=\left[c_{k}^{(1)} \gamma_{1}^{(2)}-c_{k}^{(2)} \gamma_{5}^{(2)}\right](-i)^{k} r_{I} J_{k}\left(\xi_{A} r_{I}\right) \\
\hat{\psi}_{k}^{(2)}=c_{k}^{(2)} \gamma_{4}^{(2)}(-i)^{k} r_{I} J_{k}\left(\xi_{A} r_{I}\right) \\
\hat{\psi}_{k}^{(3)}=\left[c_{k}^{(1)} \gamma_{1}^{(2)}+c_{k}^{(2)} \gamma_{5}^{(2)}\right](-i)^{k} r_{I} J_{k}\left(\xi_{A} r_{I}\right) \\
v_{k}^{(1)}=\frac{1}{2}\left(c_{k}^{(1)}+i c_{k}^{(2)}\right)(-i)^{k} r_{I} J_{k}\left(\xi_{A} r_{I}\right) \\
v_{k}^{(2)}=\frac{1}{2}\left(c_{k}^{(1)}-i c_{k}^{(2)}\right)(-i)^{k} r_{I} J_{k}\left(\xi_{A} r_{I}\right)
\end{gathered}
$$


The distinction between symmetric and antisymmetric modes occurs in the definition of the coefficients $\gamma_{j}^{(i)}$. The following equations provide their definition for the antisymmetric case. The coefficients for the symmetric mode are found analogously by interchanging sine and cosine terms whose arguments depend on the substrate half-thickness $b$, and by replacing the antisymmetric wavenumber, $\xi_{A}$, by its symmetric counterpart, $\xi_{S}$.

$$
\begin{gathered}
\gamma_{1}^{(1)}=\sin \alpha b \cos \beta b \\
\gamma_{2}^{(1)}=4 \alpha \beta \cos \alpha b \sin \beta b \\
\gamma_{3}^{(1)}=\gamma_{1}^{(1)}\left(\frac{\xi_{A}^{4}}{2}+\beta^{4}-\beta^{2} \frac{\xi_{A}^{2}}{2}\right)+\gamma_{2}^{(1)} \frac{\xi_{A}^{2}}{2} \\
\gamma_{4}^{(1)}=\gamma_{1}^{(1)}\left(\frac{3}{4} \xi_{A}^{2} \beta^{2}-\frac{\xi_{A}^{4}}{4}\right)-\frac{\gamma_{2}^{(1)}}{2} \xi_{A}^{2} \\
\gamma_{5}^{(1)}=-i \frac{\xi_{A}^{2}}{4}\left[\left(\xi_{A}^{2}-3 \beta^{2}\right) \sin \alpha b \cos \beta b+4 \alpha \beta \cos \alpha b \sin \beta b\right] \\
\gamma_{1}^{(2)}=-i \frac{\xi_{A}^{2}}{4}\left[\left(\xi_{A}^{2}-3 \beta^{2}\right) \sin \alpha b \cos \beta b+4 \alpha \beta \cos \alpha b \sin \beta b\right] \\
\gamma_{2}^{(2)}=\sin \alpha b \cos \beta b \\
\gamma_{3}^{(2)}=4 \alpha \beta \cos \alpha b \sin \beta b \\
\gamma_{4}^{(2)}=\gamma_{2}^{(2)}\left(\frac{\xi_{A}^{4}}{2}+\beta^{4}-\beta^{2} \frac{\xi_{A}^{2}}{2}\right)+\gamma_{3}^{(2)} \frac{\xi_{A}^{2}}{2} \\
\gamma_{5}^{(2)}=\gamma_{2}^{(2)}\left(\frac{\xi_{A}^{4}}{4}-\frac{3}{4} \xi_{A}^{2} \beta^{2}\right)+\frac{\gamma_{3}^{(2)}}{4} \xi_{A}^{2}
\end{gathered}
$$




\section{Acknowledgments}

This work is sponsored by the Air Force Office of Scientific Research under grant FA9550-06-1-0071. The project manager is Dr. Victor Giurgiutiu.

\section{References}

${ }^{1}$ Raghavan, A. and Cesnik, C., "Review of guided-wave based structural health monitoring," The Shock and Vibration Digest, Vol. 39, No. 2, 2007, pp. 91-114.

${ }^{2}$ Giurgiutiu, V., "Lamb Wave Generation with Piezoelectric Wafer Active Sensors for Structural Health Monitoring," Proceedings of the SPIE, Vol. 5056, 2003, pp. 111-122.

${ }^{3}$ Raghavan, A. and Cesnik, C., "Finite-dimensional piezoelectric transducer modeling for guided wave based structural health monitoring," Smart Materials and Structures, Vol. 14, No. 6, 2005, pp. 1448-1461.

${ }^{4}$ Wilkie, W., High, J., and Bockman, J., "Reliability Testing of NASA piezocomposite actuators," Proceedings of the 8th International Conference on New Actuators, Bremen, Germany, 2002.

${ }^{5}$ Bent, A. and Hagood, N., "Piezoelectric fiber composites with interdigitated electrodes," Journal of Intelligent Materials, Systems and Structures, Vol. 6, 1995, pp. 338-349.

${ }^{6}$ Williams, R., Grimsley, B., Inman, D., and Wilkie, W., "Manufacturing and Cure Kinetics Modeling for Macro Fiber Composite Actuators," Journal of Reinforced Plastics and Composites, Vol. 23, No. 16, 2004, pp. 1741-1754.

${ }^{7}$ Wilkie, W. and High, J., "Method of Fabricating NASA-Standard Macro-Fiber Composite Piezoelectric Actuators," Nasa/tm-2003-212427, NASA.

${ }^{8}$ Raghavan, A. and Cesnik, C., "3-D Elasticity-based Modeling of Anisotropic Piezocomposite Transducers for Guided Wave Structural Health Monitoring," Proceedings of the 47 AIAA Structures, Structural Dynamics and Materials Conference, 2006.

${ }^{9}$ Crawley, E. and de Luis, J., "Use of piezoelectric actuators as elements of intelligent structures," AIAA Journal, Vol. 25, 1987, pp. 1373-85.

${ }^{10}$ Raghavan, A. and Cesnik, C., "Modeling of piezoelectric-based Lamb wave generation and sensing for structural health monitoring," Proceedings of the SPIE Symposium on Smart Structures and Materials / NDE 2004, 2004.

${ }^{11} \mathrm{Cuc}, \mathrm{A}$. and Giurgiutiu, V., "Embedded non-destructive evaluation for structural health monitoring, damage detection, and failure prevention," The Shock and Vibration Digest, Vol. 37, No. 2, 2005, pp. 83-105.

${ }^{12} \mathrm{http}: / /$ www.efunda.com/materials/piezo, Engineering Fundamentals Material Database, 2007.

${ }^{13}$ Lim, J., Two-Dimensional Signal and Image Processing, Prentice Hall Signal Processing Series, 1989. 1974.

${ }^{14}$ Churchill, R., Brown, J., and Verhey, R., Complex Variables and Applications, 3rd Edition, McGraw-Hill Book Company,

${ }^{15}$ Wilcox, P., Lowe, M., and Cawley, P., "Mode and Transducer Selection for Long Range Lamb Wave Inspection," Journal of Intelligent Materials Systems and Structures, Vol. 12, 2001, pp. 553-565.

162001 ABAQUS/Standard User's Manual Version 6.2, Hibbitt, Karlsson, and Sorensen Inc., Providence, RI, 2001.

${ }^{17}$ Werwer, A., Polak, M., and Santamarina, J., "Rayleigh wave Propagation for the Detection of Near Surface Discontinuities: Finite Element Modeling," Journal of Nondestructive Evaluation, Vol. 22, No. 2, 2003, pp. 39-52.

${ }^{18}$ Alleyne, D. and Cawley, P., "The Interation of Lamb Waves with Defects," IEEE Transactions on Ultrasonics, Ferroelectrics, and Frequency Control, Vol. 39, No. 3, 1992, pp. 381-397.

${ }^{19}$ Chaudry, P. and Rogers, C. A., "The Pin-Force Model Revisited," Journal of Intelligent Materials Systems and Structures, Vol. 6, No. 3, 1994, pp. 347-354.

${ }^{20}$ Nguyen, C. H. and Kornmann, X., "A Comparison of Dynamic Piezoactuation of Fiber-based Actuators and Conventional PZT Patches," Journal of Intelligent Materials Systems and Structures, Vol. 17, No. 1, 2006, pp. 45-55.

${ }^{21}$ Lloyd, J. M., "Electrical Properties of Macro-Fiber Composite Actuators and Sensors," M.s. thesis, Virginia Polytechnic Institute and State University, 2004.

${ }^{22}$ http://www.loctite.com, Loctite Research, Development and Engineering; Technical Data Sheet for Hysol 9491, 2003. 